Nouvelles perspectives en sciences sociales

\title{
Quand des sociologues rencontrent des informaticiens : essai de formalisation, méta-modélisation, modélisation et simulation des systèmes d'action concrets
}

\section{Pascal Roggero et Christophe Sibertin-Blanc}

Volume 3, numéro 2, mars 2008

URI : https://id.erudit.org/iderudit/602476ar

DOI : https://doi.org/10.7202/602476ar

Aller au sommaire du numéro

Éditeur(s)

Prise de parole

ISSN

1712-8307 (imprimé)

1918-7475 (numérique)

Découvrir la revue

Citer cet article

Roggero, P. \& Sibertin-Blanc, C. (2008). Quand des sociologues rencontrent des informaticiens : essai de formalisation, méta-modélisation, modélisation et simulation des systèmes d'action concrets. Nouvelles perspectives en sciences sociales, 3(2), 41-81. https://doi.org/10.7202/602476ar
Résumé de l'article

Le texte résulte d'une collaboration inédite entre sociologues et informaticiens. Il présente un travail de formalisation de la sociologie de l'action organisée qui a abouti à l'élaboration d'un méta-modèle de cette théorie. Ce méta-modèle permet de modéliser des systèmes d'action concrets et de simuler leur fonctionnement. A travers l'exposition des résultats théoriques et leur application à un cas simple, nous souhaitons montrer que cette démarche présente un intérêt théorique et méthodologique pour la sociologie. Elle permet de préciser des concepts sociologiques initialement exprimés en langage naturel afin de pouvoir les évaluer de manière quantitative. Elle rend possible la simulation de situations organisationnelles et ouvre ainsi la voie à une exploration des états possibles d'un système d'action concret. Ces éléments constituent, à nos yeux, un enrichissement de la pratique sociologique. 


\title{
Quand des sociologues rencontrent des informaticiens : essai de formalisation, méta- modélisation, modélisation et simulation des systèmes d'action concrets
}

\author{
PASCAL ROggero \\ CIRESS-LEREPS, Université de Toulouse, Toulouse 1
}

ChRISTOPHE SiberTIN-BLANC IRIT, CNRS-Université de Toulouse, Toulouse $1^{1}$

"This discipline means that it is impossible to bevague about what is being assumed. It also means that the model is potentially open to inspection to other researchers to all its details. These benefits of clarity and precision also bave disadvantages, however. " Nigel Gilbert, 2004

L'expression peu amène de "sciences molles » renvoie très généralement les sciences sociales à leur défaut de formalisation et de caractère expérimental. Pourtant, depuis une quinzaine d'années l'approche dite des «systèmes complexes» se développe dans le champ de ces sciences. Mais ce développement est inégal tant sur le plan des disciplines que sur celui des aires culturelles. Puissant en économie et, à un degré moindre,

Cet article résulte d'un travail collectif soutenu par l'Action concertée incitative "Systèmes complexes en sciences humaines et sociales" du CNRS et du Ministère français de la Recherche. Outre les auteurs cités, il a principalement associé : Matthias Mailliard (IRIT), Claude Vautier (CIRESS-LEREPS), Françoise Adreit (IRIT) et Paul Chapron (IRIT). 
en sociologie dans le monde anglo-saxon, il est encore limité dans la sociologie française et, plus généralement, francophone. Si l'on peut proposer des éléments d'explication à cette réticence française - la méfiance à l'égard de la systémique parsonienne, le peu d'échos des «révolutions » cybernétiques, l'appétence limitée pour la formalisation, la reconnaissance tardive de l'individualisme méthodologique encore trop souvent confondu avec l'utilitarisme, etc. - on ne saurait s'en satisfaire durablement. En effet, dans cet ensemble encore assez hétéroclite que constituent les travaux autour des systèmes complexes, de nouveaux outils de formalisation, de modélisation et de simulation apparaissent et de nouvelles problématiques se structurent qui peuvent intéresser les sociologues. Ici un èxercice de définition, nécessairement limité et provisoire, s'impose.

Un système est perçu complexe quand son comportement ne peut pas être déduit de la connaissance du comportement des entités qui le composent. "C'est l'imprévisibilité potentielle (non calculable a priori) des comportements de ce système, liée en particulier à la récursivité qui affecte le fonctionnement de ses composants ${ }^{2}$ » qui caractérise la complexité d'un système. Pour utiliser un langage plus technique, on dira qu'un système complexe est imprévisible, que son comportement se caractérise par une "auto-éco-organisation ", c'est-à-dire une autoorganisation adaptative, que l'information y est "distribuée » entre ses composants et qu'il est capable de développer des propriétés émergentes. Nombre de phénomènes sociaux relèvent d'une telle caractérisation et c'est la raison pour laquelle les systèmes complexes ont été mobilisés pour rendre compte, entre autres, de dynamiques organisationnelles et de réseaux sociaux, de l'émergence de règles, de normes ou de formes institutionnelles, de la diffusion des opinions, ou encore de processus de ségrégation urbaine. Mais, les outils des systèmes complexes répondent aussi au besoin de traiter des données de plus en plus massives, traitement rendu possible par le recours à la puissance de calcul des ordinateurs. Enfin, avec le développement des modèles de simulation, notamment les modèles multi-agents, un champ potentiellement très fécond s'ouvre aux sociologues, autour de la simulation sociale.

Programme européen Modélisation de la complexité-Association pour la pensée complexe, Lexique, 2007, http://www.mcxapc.org, consulté le 25 juin 2007. 
Le texte présenté ici s'inscrit dans cette perspective. Il propose une tentative de formalisation d'une théorie sociologique éprouvée - la sociologie de l'action organisée ${ }^{3}$ - dans le but d'élaborer un métamodèle permettant la simulation de «systèmes d'action concrets ». Audelà de la formalisation proposée, il s'agira donc d'avancer dans le sens d'une forme d'" expérimentation virtuelle », inédite dans le domaine et permise par le recours aux systèmes multi-agents ${ }^{4}$. Notre présentation comptera cinq parties. Nous nous attacherons à définir succinctement la sociologie de l'action organisée (SAO) et à exposer plus précisément les ambitions de ce projet tant du point de vue de la sociologie que de l'informatique. Nous examinerons ensuite le méta-modèle élaboré dont nous définirons les propriétés structurelles. Enfin, nous examinerons notre simulation du comportement des acteurs d'un système d'action concret. Les trois derniers points seront exemplifiés par un cas concret élémentaire, le cas Trouville. Enfin, nous nous interrogerons, en conclusion, sur les perspectives du travail en cours.

\section{De la sociologie de l'action organisée à sa formalisation}

\section{1. Éléments de sociologie de l'action organisée}

Depuis les années 1970, l'école française de sociologie des organisations a développé un programme de recherche dont la fécondité n'est pas discutée. Ce corpus sociologique est l'un des plus enseignés en France tant aux spécialistes qu'aux non-spécialistes, notamment les futurs cadres dirigeants. Rappelons succinctement qu'il s'agit de découvrir le fonctionnement réel d'une organisation au-delà des règles formelles qui le codifient. Les organisations sont des " construits sociaux " actualisés dans et par les relations que les acteurs organisationnels entretiennent entre eux. Ces acteurs sont dotés d'une rationalité limitée et mobilisent leurs ressources pour disposer du pouvoir leur permettant de préserver et (ou) d'accroître leur autonomie et leur capacité d'action dans

3 Erhard Friedberg, Le powvoir et la règle. Dynamiques de l'action organisée, Paris, Seuil, coll. «Sociologie », 1993, 405 p.

4 Jacques Ferber, Les systèmes multi-agents. Vers une intelligence collective, Paris, InterEditions, [1995] 2007. Sur la simulation, Frédéric Amblard et Denis Phan, Modélisation et simulation multi-agents. Applications aux $S$ ciences de l'Homme et de la Société, Hermès Science Publication, 2006. 
l'organisation. Le pouvoir d'un acteur résulte de la maitrise d'une ou de plusieurs "zones d'incertitude », c'est-à-dire d'une ressource nécessaire à l'action d'autrui et dont il maitrise, au moins partiellement, l'accès. Cette maitrise lui permet à la fois de fixer, dans une certaine mesure, "les termes de l'échange" dans la relation avec autrui et de rendre son comportement plus ou moins imprévisible. Dès lors, les relations de pouvoir structurent des configurations sociales, relativement stabilisées, qualifiées de "systèmes d'action concrets» (SAC). Un SAC peut être défini comme l'ensemble constitué, dans un contexte organisationnel donné, par les acteurs et leurs alliances, leurs relations et la régulation de ces dernières. Un SAC est donc un contexte d'interaction assez précisément délimité qui structure la coopération d'un ensemble déterminé d'acteurs, de façon certes contraignante, mais sans leur ôter toute marge de manceuvre. Si, selon Friedberg, " tout contexte d'action peut être conceptualisé comme sous-tendu par un $\mathrm{SAC}^{5}$ », la finalité d'une recherche s'inscrivant dans ce corpus est bien d'identifier le $\mathrm{SAC}$ qui sous-tend toute organisation ou "action organisée ".

Avec la sociologie de l'action organisée, Friedberg a proposé d'étendre l'application du raisonnement organisationnel ${ }^{6}$ à toutes les formes d'action "organisée " quel que soit leur niveau de codification ou de formalisation. En d'autres termes, la recherche s'est déplacée vers l'identification des processus organisationnels aussi bien dans les organisations traditionnelles - fortement formalisées - que vers les actions plus floues - moins formalisées. Il proposait, de la sorte, de passer d'une sociologie spécialiséc - celle des organisations - à une sociologie générale - celle de l'action organisée.

Cependant, la sociologie de l'action organisée demeure l'héritière de la sociologie des organisations. C'est-à-dire que son domaine de prédilection reste les organisations, à savoir des ensembles assez fortement codifiés, assez précisément délimités et où les acteurs sont durablement ensemble et où il existe un objectif - celui de l'organisation partiellement différenciable des objectifs de chaque acteur.

Erhard Fricdberg, op. cit., p. 156.

" Michel Crozier et Erhard Friedberg, L'A Atur etle Système. Contraintes de l'action collectiv'e, Paris, Seuil, 1977. 


\subsection{Une ambition scientifique limitée}

Mais si cette perspective a donné lieu à de nombreuses applications empiriques de qualité, il est permis de penser qu'elle est néanmoins obérée par une stagnation à la fois théorique et méthodologique. Sur le plan théorique, les concepts mobilisés sont globalement les mêmes que ceux développés dès 1977 - système d'action concret, pouvoir, zone d'incertitude, relations, régulation - et n'ont pas fait l'objet d'un réexamen approfondi.

Sur le plan méthodologique, la sociologie de l'action organisée utilise toujours quasi exclusivement des outils de nature qualitative - entretiens semi-directifs, analyse des sources documentaires, éventuellement observation participante - et plus rarement des enquêtes par questionnaires. Dès lors, cette analyse sociologique a produit une accumulation de monographies dont la comparaison est problématique. La connaissance produite, certes utile localement, s'avère difficilement généralisable et limite la légitimité scientifique poppérienne de la démarche, ce qu'admet volontiers Friedberg ${ }^{7}$. Il apparaît pourtant que toutes les pistes n'ont pas été explorées pour dépasser ces limites d'ordre épistémologique, théorique et méthodologique, notamment celle ouverte dans la mouvance des "systèmes complexes ${ }^{8}$ ", de la formalisation et de la simulation orientée agents.

$\mathrm{Si}$ les collaborations effectives entre spécialistes des " systèmes complexes " et sociologues sont rares, les premiers se saisissent plus souvent des «objets » sociaux que les seconds ne s'emparent de leurs modèles de simulation. Il en résulte, sans doute encore trop souvent, des modélisations dont les fondements sociologiques sont mal assurés. Mais il s'agit là plus du résultat d'un défaut de communication entre spécialistes d'origines disciplinaires différentes que d'une incompatibilité fondamentale entre les contraintes de la formalisation induites par ces outils et la nature du savoir sociologique. Cette prétendue incompatibilité, quelquefois hâtivement décrétée, pourrait s'apparenter à une forme d'atonie intellectuelle et de manque d'attention portée à ce qui se

Erhard Friedberg, op. cit., p. 310.

8 Pour une discussion sur l'intérêt pour la sociologie de la pensée complexe et des systèmes complexes, voir Pascal Roggero, «Pour une sociologie d'après l.a Méthode», Communications, $\mathrm{n}^{\circ} 82,2008$, p. 143-159. 
46 WPSS, VOLUME 3, NUNÉRO2,2008

fait dans les autres sciences. Il est vrai qu'un véritable investissement cognitif, assez lourd compte tenu de la distance culturelle entre les disciplines plus formalisées où se sont développés les "systèmes complexes 》- physique statistique, in formatique ou biologie notamment - et la sociologie, s'avère nécessaire. Notre démarche témoigne de ce type de mutualisation.

\subsection{Une démarche de méta-modélisation}

Figure 1. Le phasage de la démarche

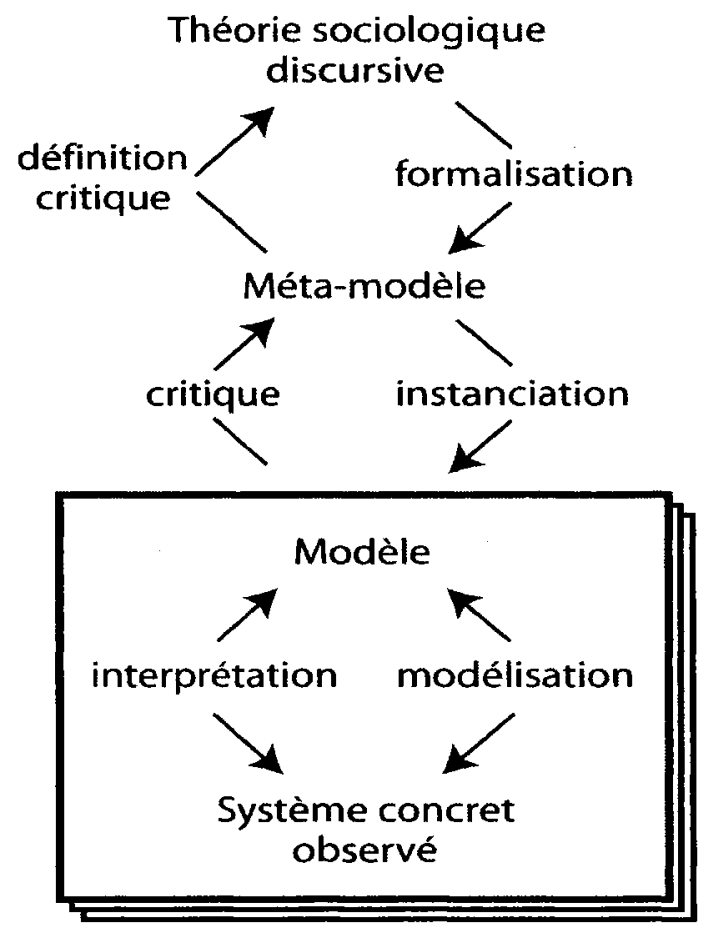

Comme le montre la figure 1 , le méta-modèle est envisagé comme une formalisation de la sociologie de l'action organisée qui se présente comme une théorie discursive. Chaque modèle est une instanciation du méta-modèle pour la modélisation d'un système particulier. La relation entre le système concret auquel nous nous intéressons et son modèle est 
des plus classiques en sociologie : elle s'appuie sur des observations qualitatives ou quantitatives de manière à élaborer, à critiquer, à affiner pour enfin valider le modèle. La relation qui relie modèle et métamodèle trouve davantage sa source dans le génie du logiciel. La définition d'un formalisme s'élabore et se valide à partir de l'accumulation des modèles qui en sont dérivés. À partir des différents cas d'études, correspondant à des contextes organisationnels différents, les modèles réalisés permettent de remettre en cause le méta-modèle et d'affiner par expériences sa définition. Enfin, le méta-modèle amène à critiquer la théorie discursive, non seulement au cours de l'étape de formalisation qui nécessite de lever les ambiguités et de préciser les concepts pour pouvoir les opérationnaliser, et même de faire apparaître de nouveaux concepts, mais aussi, avec le retour d'expérience, au regard des cas d'études. Ces derniers aspects sont plutôt originaux dans le cadre de la simulation sociale.

Examinons maintenant plus précisément notre formalisation des concepts de la sociologie de l'action organisée et le méta-modèle qui en résulte.

\section{Méta-modèle du système d'action concret et formalisation des concepts}

Le méta-modèle, c'est-à-dire le modèle général de la théorie, résulte d'une articulation logique des concepts formalisés. Nous présenterons ce méta-modèle pour donner d'entrée une vision d'ensemble au lecteur avant de détailler la formalisation des concepts de la sociologie de l'action organisée.

\subsection{Méta-modèle du système d'action concret ${ }^{9}$}

Procédant par éliminations successives, nous avons proposé comme noyau structurant de la théorie les trois constituants suivants : les ressources ou zones d'incertitude, les relations et les acteurs. Cette réduction pourrait paraître abusive, mais cette sociologie est bien une sociologie

" Pascal Roggero et Christophe Sibertin-Blanc, «Pour une formalisation de la Sociologie de l'Action Organisée ", Communication au XVII Congrès international des sociologues de langue frangaise, Tours, 5-9 juillet 2004. 
d'acteurs entretenant des relations en manipulant des ressources. Comme l'écrit Friedberg: "pas de pouvoir sans relation, pas de relation sans échange ${ }^{10} \%$. Le pouvoir suppose donc la relation qui implique l'échange qui, lui-même, nécessite des objets d'échange : des ressources.

Figure 2. Le méta-modèle du système d'action concret

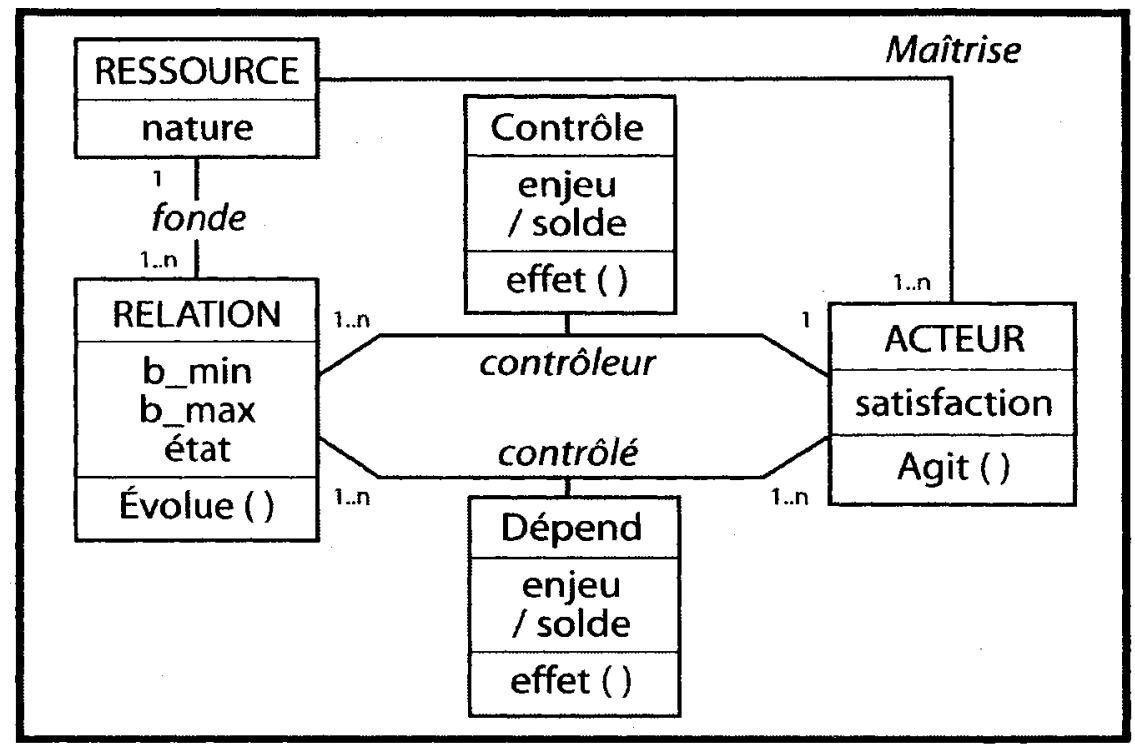

La figure 2 présente le méta-modèle de la structure des systèmes d'action concrets, dont les éléments constitutifs sont des acteurs, des ressources et de relations, reliés par les associations contrôle, dépend et maitrise. Ainsi, une ressource est le fondement, ou support, d'une ou de plusieurs relations dans chacune desquelles interviennent des acteurs, un qui la contrôle et d'autres qui en dépendent. Chaque acteur place des enjeux et se voit attribuer un solde pour chaque relation dans laquelle il est impliqué. L'acteur qui contrôle une relation en définit l'état, c'est-à-dire qu'il fixe les "termes de l'échange » de cette relation à l'intérieur d'un espace de choix éventuellement limité par l'existence de contraintes $: b_{m i n}$ pour la borne inférieure et $b_{\max }$ pour la borne supérieure. En agissant sur l'état d'une relation, l'acteur qui la contrôle décide de la manière dont

1" Erhard Friedberg, op. cit., p. 115. 
sont distribués les soldes entre les acteurs compte tenu de leur fonction d'effet qui définit, pour chaque relation et chaque acteur, le solde reçu par ce dernier en fonction de l'état de la relation. Chaque acteur maitrise une ou plusieurs ressources. Après cet aperçu général, il convient d'examiner les choses plus en détail.

\subsection{Formalisation des concepts de la sociologie de l'action organisée}

Commençons par les constituants du méta-modèle, avant de voir ce qui se joue dans les relations, sous quelles contraintes et présenter enfin notre formalisation du pouvoir et de la satisfaction.

\subsubsection{Constituants du méta-modèle : ressources, acteurs et relations ${ }^{11}$}

Les ressources d'un système d'action concret sont, dans le sens le plus large du terme, les éléments nécessaires à l'action organisée dont la disponibilité est requise pour réaliser une certaine action. Chaque ressource possède une propriété nature, dont la valeur est l'une des quatre sources de pouvoir identifiées par la sociologie de l'action organisée : une compétence difficilement remplaçable, la maîtrise de relations avec l'environnement du système, la maîtrise de l'information et de la communication interne et, enfin, l'utilisation stratégique des règles organisationnelles à l'occasion des marchandages auxquels elles donnent lieu.

Toute ressource est maîtrisée par un ou plusieurs acteurs; maîtriser une ressource c'est être en situation de la rendre plus ou moins disponible et ainsi influer sur la capacité d'action des acteurs qui en ont besoin.

Chaque ressource donne lieu à un certain nombre de relations. Une relation correspond à un certain type de transactions concernant la ressource sur laquelle elle est fondée, et elle est déséquilibrée : un (unique) acteur - l'un de ceux qui maitrisent la ressource - contrôle cette relation, tandis que d'autres acteurs - ceux qui ont besoin de cette ressource pour atteindre leurs objectifs - sont contrôlés, dominés, ou

11 Nous écritons dans cette partie ces termes en italique afin de bien spécifier qu'il s'agit des entités du méta-modèle qu'on ne peut confondre avec ce qu'elles sont censées représenter, le système concret. Les entités de base du méta-modèle commencent en plus par une majuscule. 
encore, dépendants dans cette relation. En effet, c'est l'acteur qui contrôle la relation qui détermine dans quelle mesure la ressource est accessible et qui ainsi contrôle la possibilité pour les acteurs dépendants de réaliser les actions liées à leurs objectifs. L'idée que la relation de pouvoir est toujours déséquilibrée est centrale dans la sociologie de l'action organisée. Il n'y a donc pas de problème à considérer qu'il y a toujours un acteur en position de force dans la relation ${ }^{12}$. Mais cette relation est aussi réciproque, le dominé a toujours les moyens de monnayer, dans une certaine mesure, sa collaboration. Si nous raisonnons dans notre modélisation sur une seule relation nous ne prenons pas en compte cette dimension réciproque, elle est cependant intégrée dès que nous considérons l'ensemble des relations existant entre les acteurs. Ainsi un acteur $a$ dominé par $b$ dans une relation peut dominer $b$ dans une autre relation et compenser sur cette relation sa faiblesse sur l'autre. Reste que si $a$ ne contrôle aucune autre relation où $b$ est impliqué, nous ne savons pas prendre en compte, en l'état actuel de notre modèle, cette réciprocité.

Tout acteur maitrise une ou plusieurs ressources; c'est donc reconnaître à tout acteur d'un système d'action concret une certaine marge de manœuvre, celle qu'il exerce sur les ressources qu'il contrôle et, par là même, dénier le statut d'acteur social à toute personne qui ne maitriserait aucune ressource. L'exercice effectif de la maitrise d'une ressource passant nécessairement par le contrôle de l'une des relations qu'elle fonde, chaque acteur contrôle au moins une relation. Cela ne contrevient pas à la sociologie de l'action organisée qui postule que chaque acteur, quelle que soit sa position, a la possibilité d'exercer sa maitrise sur certaines ressources, fussent-elles mineures. Tout acteur maitrise donc au moins une relation, et c'est cette maitrise qui lui confère son statut d'acteur social. De façon dialogique, les notions de ressource et d'acteur se définissent l'une par rapport à l'autre : une ressource n'est telle que dans le mesure où certains acteurs en dépendent pour réaliser leur activité; est acteur celui qui contrôle une ressource.

Il en résulte que, dans la modélisation d'un système d'action concret, la correspondance entre acteur et personne physique n'a rien d'automatique. Ainsi, les individus qui ne maîtrisent aucune relation ne

Erhard Friedberg, op. cit., p. 113. 
sont pas des acteurs. Certains individus peuvent donner lieu à plusieurs acteurs s'ils n'opèrent aucune interférence entre différentes fonctions qu'ils exercent, en totale indépendance les unes des autres. A contrario, une population homogène peut être agrégée en un acteur unique si chacun de ses membres est en situation de dépendance et de contrôle vis-à-vis des mêmes relations (et ce, avec les mêmes enjeux), si bien qu'ils auront des comportements similaires. Enfin, un groupe, une assemblée ou une coalition peut constituer un acteur collectif si seul le comportement résultant de l'ensemble a un impact. C'est donc bien l'analyse sociologique qui identifie les acteurs du système d'action concret, à un niveau de granularité qui dépend de la finalité de cette analyse. La seule caractéristique constitutive d'un acteur est d'être en situation de dépendance ou de contrôle vis-à-vis de relations bien identifiées et d'être capable d'exercer ce contrôle de façon finalisée.

\subsubsection{Ce qui se joue dans la relation : enjeux et soldes}

Considérer un acteur comme stratégique revient à lui attribuer un comportement intéressé, c'est-à-dire "motivé par une visée, sans préciser davantage la nature de cette visée ou de ce mobile ${ }^{13} »$. Cette visée l'amène à mobiliser les moyens dont il dispose pour tenter d'atteindre cette dernière et ainsi à utiliser les relations dans lesquelles il est impliqué. Chacune de ces relations aura plus ou moins de valeur à ses yeux, c'est ce que traduit la notion d'enjeu. Chaque acteur répartit donc des enjeux sur chacune des relations auxquelles il participe, que ce soit en tant que contrôleur de cette relation ou en tant que dépendant ou contrôlé. Il opère cette répartition en fonction de l'importance de la ressource correspondante vis-à-vis de sa visée ou de ses objectifs propres : plus l'usage de la ressource accessible via la relation est nécessaire pour atteindre un objectif qui est important pour l'acteur, plus l'enjeu que l'acteur place sur cette relation est élevé. Nous notons enjeu $(r, a)$ (ou Enjeu $(r, a)$ ?), l'enjeu que place l'acteur $a$ sur la ressource $r$.

13 Ibid.., p. 214-215. 
Figure 3. La visée dans la formalisation du système d'action concret ${ }^{14}$

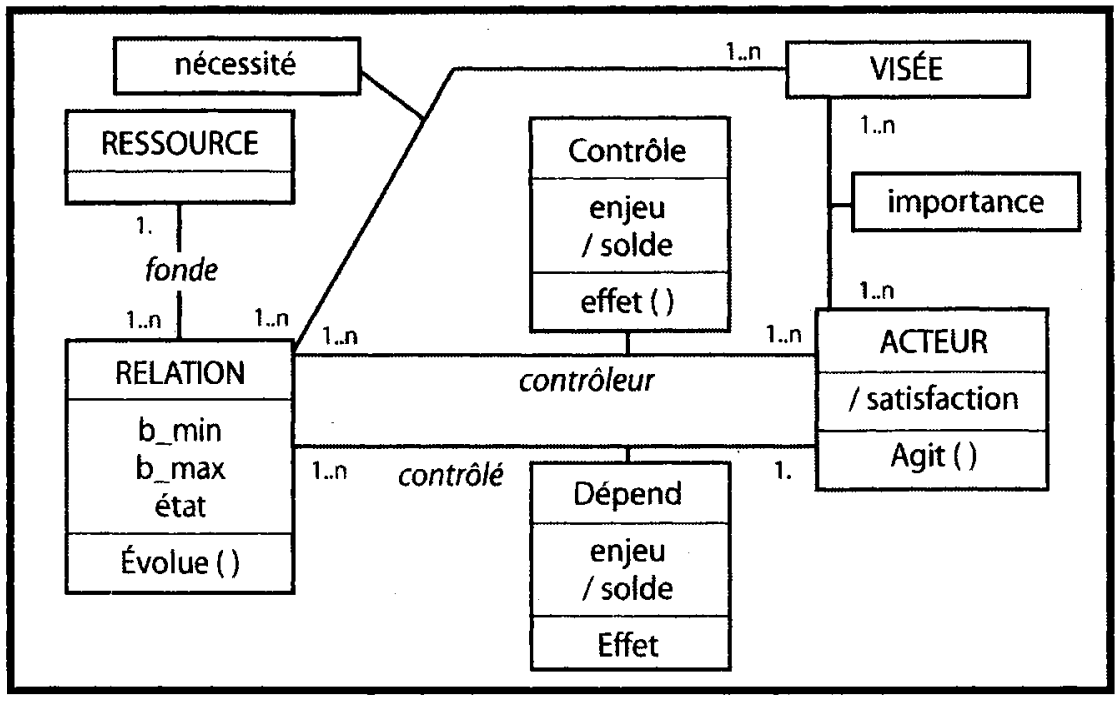

Pour un acteur et une relation donnés, la valeur de la propriété enjeu des associations contrôler ou dépendre est déterminée par la valeur des propriétés importance et nécessité. Cette répartition des enjeux d'un acteur correspond à l'impact opérationnel de sa visée ou de ses objectifs sur son comportement. Pour l'analyse du fonctionnement d'un système d'action concret, ce qui importe ce n'est pas tant la nature de la visée ou des objectifs d'un acteur que ce qu'ils le conduisent à réaliser. Les enjeux sont le maillon qui, conformément à l'hypothèse de rationalitét ${ }^{15}$ des acteurs, permet de relier causalement le comportement d'un acteur avec ses objectifs. Il s'agit bien du mode de raisonnement de la sociologie de l'action organisée même si Friedberg insiste sur la relation récursive entre préférences (ou objectifs) et comportements ${ }^{16}$. L'acteur qui

4 L'entité ressource n'y est pas montrée pour ne pas surcharger le diagramme.

15 Que Friedberg (op. cit.,p. 214) qualifie de « située " pour insister sur les limitations que Simon a mises en lumière et les faire dépendre ou les inscrire dans le contexte d'action. Nous reviendrons plus loin sur la discussion de cette hypothèse de rationalité.

14 Il discute utilement la relation univoque des préférences déterminant les choix que les économistes présupposent en mobilisant Festinger, March et Simon, Lindblom ou Weick, mais cela reste un peu décoratif dans la mesure où, par la suite, il n'utilise 
contrôle une relation est celui qui détermine les «termes de l'échange » de la ressource qu'il possède, c'est-à-dire les conditions selon lesquelles les tractations, les marchandages concernant l'accès à la ressource se déroulent et, surtout, se concluent. Friedberg précise que le pouvoir se définit comme "la capacité d'un acteur à structurer des processus d'échange plus ou moins durables en sa faveur, en exploitant les contraintes et les opportunités de la situation pour imposer les termes de l'échange favorables à ses intérêts ${ }^{17}$ ". Il ajoute que " [ce processus] structuré [s] de telle sorte que tous les participants en retirent quelque chose, tout en permettant à quelqu'un (ou quelques-uns) d'en retirer davantage que les autres ${ }^{18}$ ».

Ce que les acteurs "retirent" de l'échange, nous l'exprimons par le terme de solde. Bien qu'assez fruste et non dénué d'une connotation comptable un peu réductrice, ce vocable se distingue de celui de " termes de l'échange " dans la mesure où il évoque le résultat de la transaction et non les règles qui président à son déroulement. C'est donc l'acteur qui contrôle une relation qui fixe la valeur du solde pour chacun des acteurs participant à cette relation, c'est-à-dire lui-même et tous les acteurs dépendants dans cette relation. Ce solde correspond à la qualité de la disponibilité de la ressource; plus ou mieux la ressource sera utilisable par un acteur, plus son solde pour cette relation sera élevé.

Le critère de répartition des soldes entre les différents participants d'une relation est spécifique à chaque relation. On s'attend à trouver des relations où les soldes sont " à somme nulle ", ou du moins se compensent : si l'usage de la ressource est bon pour certains acteurs, il le sera d'autant moins pour les autres. D'autres relations, dans lesquelles le solde du contrôleur et celui des acteurs dépendants varient dans le même sens, peuvent être qualifiées de gagnant-gagnant ou de perdantperdant selon que ces soldes ont tendance à être favorables ou défavorables. La distribution des soldes et des enjeux sur des échelles de valeurs numériques, et non pas seulement qualitatives, permet, par l'application d'opérations simples, d'agréger ces valeurs en des grandeurs synthétiques et interprétables sociologiquement.

plus cet en richissement, Ibid., p. 50.

17 Ibid., p. 117-118.

is Ibid., p. 118. 


\section{NPS\$, VOUUME S, NUNERO 2, 2008}

Nous pouvons graduer les enjeux sur une échelle:

$n u l=0$, négligeable $=1, \ldots$ important $=5, \ldots$ vital $=10$,

ce qui signifie aussi que nous devons attribuer aux acteurs une quantité maximale et unique d'enjeux, ici fixée à 10 .

Sur le plan théorique, nous pouvons discuter cette condition d'une quantité maximale et unique d'enjeux pour tous les acteurs. Néanmoins, elle ne nous semble pas contrevenir aux hypothèses de la sociologie de l'action organisée. En effet, Friedberg écrit qu' « aucun acteur d'un système (construit comme tel par l'analyste) ne peut y être sans intérêt et sans enjeu ${ }^{19}$ ". Il propose de distinguer l'« engagement dans le jeu » et les "préférences " pour le jeu, c'est-à-dire avoir des intérêts dans le jeu. Il est possible d'être peu engagé dans le jeu - comme les ouvriers retraitistes ${ }^{20}$, vivre pour les loisirs -, mais il est impossible de ne pas y avoir un intérêt. Pour être en mesure de bien se désengager du jeu, il faut jouer le jeu pour obtenir, par exemple, le maximum d'arrangements d'horaires, des "entorses " au règlement, etc. Donc, nous nous plaçons bien ici sur le plan des "préférences " et non sur le plan de l'« engagement " pour le jeu. Dans ce cadre, notre quantité d'enjeux égale pour chacun des acteurs n'est pas infondée.

Les relations font l'objet d'une fixation des "termes de l'échange " par l'acteur contrôleur dans un espace limité par des contraintes.

2.2.3. Contraintes sur les « termes de l'échange » des relations et sur les fonctions d'effet des acteurs

Il nous reste à donner l'interprétation de la propriété contrainte figurant dans les relations; elle est n'est pas explicitement pensée mais juste citée dans le corpus théorique de la sociologie de l'action organisée. Cependant, elle nous semble indispensable à la modélisation du fonctionnement d'un système d'action concret. En effet, l'acteur qui contrôle une relation ne peut pas pour autant attribuer n'importe quelle valeur aux soldes des acteurs participants à cette relation. Il doit respecter « les règles du jeu social » qui déterminent, en partie, l'espace

19 Ibid., p. 267.

21. Renaud Sainsaulieu, L'identité au travail, Paris, Presses de Science Po, [1977] 1988. 
des valeurs qu'il peut donner aux soldes des acteurs de la relation, y compris lui-même. L'origine de ces contraintes est soit institutionnelle de par les règles formelles internes ou imposées à l'organisation (par exemple, la nature des contrats de travail légaux), soit technique ou de faisabilité de par l'impossibilité physique tenant à la nature de la ressource (on ne peut donner moins d'information que pas d'information du tout... sauf à verser dans la désinformation), soit encore normative tenant à l'acceptabilité sociale en fonction des normes en vigueur. Nous proposons de formaliser les contraintes associées à une relation de la manière suivante:

- deux valeurs : $b_{\min }$ et $b_{\max } \quad$ telles que $:-1 \leq b_{\min }<b_{\max } \leq 1$;

- tout acteur a participant à la relation $r$ a une fonction qualifiée de fonction d'effet ainsi définie : Effet $t_{(r, a)}:[-1,1] \longrightarrow[-10,10]$.

Cette relation détermine le solde obtenu par l'acteur a compte tenu de la valeur qu'il fixe pour les «termes de l'échange », puisqu'il est ici contrôleur de la relation $r$. Explicitons cette formalisation.

L'intervalle $[-1,1]$ correspond à l'espace de choix de l'acteur contrôleur quand il dispose d'une totale maîtrise de la relation. Ainsi, en choisissant une valeur dans cet intervalle, il définit les " termes de l'échange » ou encore la facilité d'accès à la ressource qu'il accorde aux autres acteurs.

Le choix (par l'acteur contrôleur) d'une valeur $a \in[-1,1]$ produit l'Effet $t_{(r, a, a)}$, c'est-à-dire la valeur du solde de l'acteur $a$ (ce qu'il « retire de la relation »). Il est possible d'utiliser tout autre nombre pour borner l'espace de choix, le choix des valeurs -1 et +1 n'a pas d'incidence sur les résultats d'une modélisation. Seule compte ici la façon dont les différentes fonctions sont définies pour chacun des acteurs participant à la relation. En effet, ces fonctions d'effet prennent des formes spécifiques pour chaque acteur. Elles définissent la manière dont les soldes de chaque acteur d'une relation évoluent en fonction des valeurs prises par les "termes de l'échange » de cette même relation. Comme nous le verrons dans notre exemple, nous avons recouru à des formes mathématiques simples, linéaires ou hyperboliques. Dans le cadre des études empiriques que nous aurons à mener ultérieurement, il faudra s'interroger là aussi sur les moyens d'éclairer la forme que peuvent 
prendre les fonctions d'effet des acteurs concrets, en essayant ensuite de trouver la forme mathématique la plus approchante. Le cas traité plus loin n'a pas permis de le faire et nous nous sommes contentés d'inférer les fonctions d'effet de données assez succinctes (cas pédagogique simple).

Quant aux valeurs $b_{\min }$ et $b_{\max }$, elles permettent de rendre compte du fait que l'acteur contrôleur ne peut pas fixer n'importe quelle valeur de l'espace de choix théorique $[-1,1]$ et que sa maitrise de la relation ne peut s'exercer que dans un intervalle seulement de cet espace de choix. L'écart ou la distance $\left(b_{\max }-b_{\text {min }}\right)$ de l'intervalle $\left[b_{\text {min }}, b_{\text {max }}\right]$ représente donc le niveau de maîtrise effective de l'acteur contrôleur sur la relation.

Le codage numérique des valeurs prises par les soldes permet d'exprimer de manière concise les contraintes qui s'appliquent aux soldes d'une relation. Cette expression numérique est certes concise et permet ensuite une formalisation rigoureuse des concepts de la sociologie de l'action organisée, mais elle pose des problèmes d'évaluation empirique. Il est clair qu'il y a, même dans le cas d'une très bonne connaissance empirique d'un système d'action, une difficulté à apprécier précisément $\left(b_{\max }-b_{\text {minn }}\right)$, c'est-à-dire l'espace des choix possibles de l'acteur contrôleur de la relation. Et c'est la même chose pour les autres paramètres. Nous n'échappons pas à une forme d'arbitraire. Mais, sauf cas d'erreur majeure dans l'appréciation de la valeur de ces paramètres et qui correspondrait en fait à une erreur sociologique, il est possible de relativiser l'impact de l'imprécision des valeurs fixées en n'interprétant les résultats simulés du modèle comme étant significatifs que dans une fourchette d'imprécision équivalente de l'ordre de 20 à $30 \%$. Cependant, cette formalisation posée, l'enquête de térrain doit se donner les moyens d'évaluer avec le plus de fiabilité possible la valeur des paramètres. À ce propos, Nigel Gilbert indique : "Simulations [...] involve the estimation of many parameters and adequate data for making the estimates can be difficult to come by ${ }^{21} »$. Nous n'avons pas encore fait d'enquête postérieure à l'élaboration de cette formalisation et dédiée à son application, cela viendra bientôt car c'est maintenant possible. Jusqu'ici notre base empirique s'est limitée à des cas pratiques enseignés en sociologie des organisations. Voyons

${ }^{21}$ Nigel Gilbert, «Using computer simulation to study social phenomena », Bulletin de Métbodologie Sociologique, $\mathrm{n}^{\circ}$ 47, 1995, p. 102. 
maintenant comment nous avons formalisé la satisfaction et le pouvoir de l'acteur.

\subsubsection{Satisfaction et pouvoir d'un acteur}

L'état d'une organisation est défini comme l'état de chacune de ses relations nommé $s$, une grandeur particulièrement significative est alors, pour chaque acteur, le cumul, sur l'ensemble des relations auxquelles il participe, d'une combinaison de son enjeu avec le solde qui lui est attribué. Nous l'appellerons la satisfaction d'un acteur (de préférence au terme couramment employé d'utilité, en ce qu'il est plus évocateur d'une rationalité limitée); elle reflète la possibilité de l'acteur d'accéder aux ressources dont il a besoin pour atteindre ses objectifs, pondérée par son utilité pour ces ressources. De ce fait, elle mesure, pour un acteur, sa possibilité d'action en disposant des moyens nécessaires à la réalisation de ses objectifs. Il s'agit d'une définition originale qui ne se confond pas avec l'utilité des économistes.

Une version très simple et linéaire de cette satisfaction est de considérer la somme, pour toutes les relations auxquelles un acteur participe, du produit des enjeux qu'il place sur cette relation par son solde :

$\operatorname{Satis}(a, e)=\sum_{r \in R} \operatorname{enjeux}(a, r) * \operatorname{effet}\left(a, e_{r}\right)^{22}$

Le caractère stratégique du comportement de l'acteur de la sociologie de l'action organisée le conduit, par définition, à chercher à atteindre ses objectifs ou sa visée et donc le méta-objectif commun à tous les acteurs

\footnotetext{
Nous verrons plus loin que l'Effet peut aussi être caractérisé comme le powvoir potentiel dont un acteur dispose sur un ou tous les autres. Toutefois, la satisfaction d'un acteur peut dépendre de celle d'un (ou de plusieurs) autre acteur. Il s'agit de traduire ici le concept d'alliance. Nous avons donc introduit dans le calcul de la satisfaction d'un acteur cette dimension de la solidarité en l'exprimant par des coefficients de solidarité. Ces coefficients mesurent dans quelle mesure un acteur a prend en compte dans sa propre satisfaction celle des acteurs dont il est solidaire positivem ent (coefficient compris entre 0 et 1 ) ou négativement (coefficient compris entre -1 et 0 ). En notant solidarité $(a, b)$ la solidarité d'un acteur $a$ envers un acteur $b$, la satisfaction devient donc: Satis $(a, e)=$ $\sum_{b \in A}$ solidarité $(a, b)^{*} \sum_{r \in \mathrm{R}}$ enjeux $(b, r) *$ Effet $(b, e)$ avec $\forall \mathrm{a} \in A, \forall \mathrm{b} \in \mathrm{A},-1 \leq$ solidarité $(a, b) \leq 1$ et $\forall a \in A, \sum_{b \in A}$ solidarite $(a, b)=1$.
} 
sociaux : obtenir les moyens de ses objectifs, à savoir une valeur acceptable (à défaut de l'optimum) pour sa satisfaction. Un SAC apparaît alors comme étant un jeu, dans lequel le comportement des acteurs est déterminé par la satisfaction qu'il obtient en fonction de l'état de ce jeu. Cependant, un tel jeu social se distingue des jeux considérés en économie en ce que l'objectif n'est pas du tout de maximiser la satisfaction (cumulée) des acteurs, mais d'atteindre un état stationnaire. Dans un tel état du jeu, chacun a une satisfaction acceptable et donc ne cherche pas à modifier l'état des relations qu'il contrôle, les interactions entre les acteurs sont équilibrées, et donc régulées.

Une autre grandeur très significative à considérer est la mesure dans laquelle un acteur contribue à la satisfaction d'un autre, c'est-à-dire la quantité de capacité d'action qu'il lui prodigue. C'est ce qui nous semble le mieux exprimer la notion de pouvoir qui est au coeur de la sociologie de l'action organisée. Nous pouvons alors quantifier le pouvoir qu'un acteur $c$ exerce sur un acteur $b$ dans un état du système d'action $e$ de la façon suivante :

Pouvoir $(c, b, e)=\sum_{r \in \mathrm{R}}$ ic controike $r$ enjeux $(b, r){ }^{*}$ effet $t_{r}\left(b, \mathrm{e}_{\mathrm{r}}\right)^{23}$

Mais nous verrons dans la section suivante qu'il est possible d'approfondir cette question du pouvoir. Appliquons maintenant sur un exemple notre méta-modèle.

\subsection{Le méta-modèle appliqué au cas Trouville}

En guise d'illustration, nous proposons de rendre compte d'un cas très simple de fonctionnement organisationnel : le cas Travel-Tours emprunté à Patrick Smets ${ }^{24}$.

Travel-Tours est un tour operator dont deux agences, TRO1 et TRO2, sont situées dans la même ville, Trouville. Depuis quelques mois, les résultats de l'agence TRO1 s'accroissent alors que ceux de TRO2

23. Si nous intégrons les liens de solidarité entre les acteurs, ce que nous ne ierons pas dans l'illustration, le pouvoir de $c$ sur $b$ dans un état $e$ s'exprime de la manière

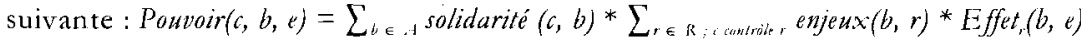

2.4 Site de Patrick Smets, L'agence Travel-Tours (Trouville), 2004, http://homepages.ulb.ac.be/ psmets1/travel.pdf, consulté en juin 2004. 
stagnent, voire régressent. Le directeur régional décide de récompenser l'équipe de TRO1 en proposant d'en titulariser la secrétaire jusque là à mi-temps dans les deux agences sur des contrats à durée déterminée. On s'attend à ce que tant le directeur que la secrétaire se réjouissent de cette proposition; or, tous deux la refusent fermement. Comment expliquer ce double refus ? En identifiant les «zones d'incertitude » et les ressources associées au sein de TRO1, une analyse en termes de SAO permet de montrer qu'aussi bien le directeur que la secrétaire ont rationnellement raison de s'opposer à cette proposition qui, si elle se concrétisait, induirait pour chacun d'entre eux une perte de pouvoir. En effet, une étude plus attentive du système relationnel révèle que :

- l'agence TRO2 est plus inventive que TRO1 en matière de conception de produits touristiques alors que TRO1 dispose d'une équipe commerciale très efficace. Travaillant à mi-temps au sein de TRO2, la secrétaire informe le directeur de TRO1 des projets de TRO2 si bien que TRO1 utilise à plein ces informations avec son équipe commerciale. - Pour des raisons personnelles, l'obtention d'un emploi stable ne fait pas partie des objectifs de la secretaire, à court terme au moins. Par contre, elle entretient de bonnes relations avec les employés de TRO1 et apprécie la situation dans laquelle elle se trouve, où les directeurs de TRO1 et TRO2 n'ont pas la possibilité d'exercer un contrôle précis sur son travail. Aussi, si la proposition de la titularisation à temps complet à TRO1 se concrétisait, d'une part, elle perdrait cette relative liberté dans son travail (ce qu'elle ne veut pas) et, d'autre part, le directeur perdrait les informations qu'elle lui fournit sur TRO2 (ce qu'il souhaite éviter).

Il s'agit de comprendre le comportement du directeur et de la secrétaire qui sont les acteurs du système de relations qui les unit. Chacun dispose d'une ressource : "l'information sur TR02 » pour la secrétaire et le «travail de la secrétaire » pour le directeur. Cette dernière fonde deux relations différentes : d'une part, la relation concernant " la stabilité dans l'emploi » de la secrétaire, et; d'autre part, celle relative au " contenu du travail » de celle-ci. La valeur des enjeux traduit les souhaits de chacun des acteurs, sachant que chacun a une somme de 10 points d'enjeux à répartir sur les trois relations. Ainsi la secrétaire, peu gênée par son statut précaire ( 2 sur la stabilité) - et peu concernée par la diffusion de l'information (1) - privilégie-t-elle le contenu de son travail (7). Le directeur, quant à lui, place la majeure partie de ses enjeux 
sur la diffusion de l'information, vitale pour lui (7), sans pouvoir se désintéresser de ce qu'il fait faire à sa secrétaire (2) et tout en négligeant la question de son statut (1).

La valeur de $b_{\max }$ et de $b_{\min }$ et la définition des fonctions d'effet nécessitent plus d'explications. Concernant « la stabilité dans l'emploi », le directeur ne dispose que d'une maîtrise partielle de la relation. En effet, s'il peut renouveler le contrat temporaire de la secrétaire, il n'est pas dans ses attributions de lui offrir un contrat définitif ce que seul le directeur régional peut faire; aussi $b_{\text {max }}$ est-il fixé à un niveau nettement inféricur à 1 , c'est-à-dire 0,4 . Sur le plan de ses possibilités de durcir la relation, le directeur est aussi contraint : il doit, outre le respect de la législation du travail, justifier sa décision auprès de sa hiérarchie et prendre en compte les réactions éventuelles des autres salariés en cas d'injustice caractérisée; il en résulte $b_{\text {min }}=-0,4$. Avoir un emploi stable produit un plein effet pour la secrétaire, c'est-à-dire que si elle l'a : Effet(sec, 1$)=10$, dans le cas contraire : Effet(sec, -1)= - 10, - 10 et 10 étant les valeurs extrèmes des soldes. Quant au directeur, sa préoccupation pour cet emploi est en proportion avec sa stabilité, mais elle reste limitée, d'où Effet(dir, $x)=3 * x$.

Le directeur dispose d'une plus grande marge de manoeuvre en ce qui concerne le contenu de l'emploi de la secrétaire. Nous interprétons les valeurs négatives dans l'espace de choix comme un contrôle strict de la quantité et de la qualité du travail réalisée par la secrétaire et de la façon dont elle s'organise, et les valeurs positives comme l'absence d'un tel contrôle. La valeur $b_{\text {min }}=-0,4$ provient de l'importance que le directeur accorde à la cordialité de ses relations avec les employés; cependant, sa fonction le rend responsable de la production de l'agence et donc de ce que réalise chaque employé, donc $b_{\max }=0,4$. L'effet sur la secrétaire est totalement proportionnel au niveau de ce contrôle, car tout employé se trouvera bien de n'avoir pas à subir un contrôle étroit de son travail d'où Effet(sec, $x)=-7^{*} \mathrm{x}$. Quant au directeur, la fonction Effet $($ dir, $x)=-3 *$ $\mathrm{x}^{2}$ correspond à l'idée que tout excès dans l'exercice de ce contrôle ne peut que lui attirer des difficultés, et que le plus confortable pour lui est d'exercer un contrôle modéré.

Concernant enfin l'information sur l'activité de l'agence TRO2, nous interprétons les valeurs positives de l'espace de choix comme la divulgation d'in formations, les valeurs négatives comme de la désinformation et la valeur nulle comme la discrétion de la secrétaire à propos de ce 
qu'elle peut savoir de l'activité de TRO2. Les valeurs proposées pour $b_{\text {min }}$ et $b_{\max }$ correspondent à la quantité d'informations dont la secrétaire peut disposer et rendre crédible auprès du directeur de TRO1. L'effet sur le directeur correspond à la pleine exploitation qu'il fera de cette information, d'où Effet(dir, $x)=10 * x$. Quant à la secrétaire, sa tranquillité lui commanderait de ne diffuser aucune information, qu'elle soit exacte ou fausse, car toute autre situation présente des risques, d'où Effet $($ sec, $x)=-2 * \mathbf{I} \times \mathbf{I}$.

Tableau 1. Enjeux, fonctions d'effet et contraintes associés aux relations

\begin{tabular}{|l|l|c|c|c|}
\cline { 2 - 5 } \multicolumn{2}{c|}{} & $\begin{array}{c}\text { Stabilité de } \\
\text { l'emploi }\end{array}$ & $\begin{array}{c}\text { Contenu } \\
\text { du travail }\end{array}$ & $\begin{array}{c}\text { Information sur } \\
\text { TRO2 }\end{array}$ \\
\hline \multirow{2}{*}{ Enjeux } & Directeur & 1 & 2 & 7 \\
\cline { 2 - 5 } & Secrétaire & 2 & 7 & 1 \\
\hline \multirow{2}{*}{ Effet } & Directeur & $3 * x$ & $-3 * x^{2}$ & $10 * x$ \\
\cline { 2 - 5 } & Secrétaire & $10 * x$ & $-7 * x$ & $-2 *|x|$ \\
\hline$b_{\text {min }}, \mathbf{b}_{\max }$ & & $-0.4,0.4$ & $-0.4,0.4$ & $-0.3,0.7$ \\
\hline
\end{tabular}

Les grandeurs que nous venons de définir et d'exemplifier permettent de calculer ce que nous appelons les propriétés structurelles du métamodèle.

\section{Propriétés structurelles d'un SAC : pertinence, autonomie et pouvoir}

Poursuivant notre travail de formalisation, il est possible de définir formellement des concepts que la sociologie de l'action organisée ne traite que de manière littéraire, notamment la pertinence des relations, l'autonomie et le pouvoirpotentieldes acteurs. Plus précisément, la définition d'un SAC comme une structure mathématique particulière permet de définir un ensemble de grandeurs qui s'avèrent significatives pour le 
sociologue et peuvent être interprétées dans les termes de la sociologie de l'action organisée.

Notre démarche permet de définir la pertinence des relations, l'autonomie et le pouvoir potentiel des acteurs à partir des éléments formalisés et quantifiés précédemment. Or, il s'agit de concepts centraux de la sociologie de l'action organisée. Examinons d'abord la pertinence des relations.

La première source du pouvoir, selon Friedberg, réside dans " la pertinence des possibilités d'action ${ }^{25} "$ qu'un acteur contrôle. Comment avons-nous formalisé cette "pertinence des possibilités d'action »? Dans notre vocabulaire, il s'agit d'évaluer la pertinence d'une ressource et donc de la ou des relations qu'elle fonde. Cette pertinence va dépendre de l'importance que les acteurs vont lui accorder. Plus la ressource est pertinente, plus les acteurs qui en ont besoin vont la considérer comme importante. Nous disposons pour exprimer cela de la notion d'enjeu: la valeur que la ressource et les relations auxquelles elle donne lieu ont pour l'acteur. Dès lors, la pertinence d'une relation $r$ peut être évaluée comme la somme des enjeux que les acteurs participant à cette relation placent sur elle:

Pertinence $(r)=\sum_{a \in A}$ enjeux $(a, r)$

Quant à l'autonomie des acteurs, elle va de pair avec leur dépendance. Si « la coopération suppose toujours une dépendance mutuelle " entre les acteurs, cette dépendance est néanmoins " déséquilibrée ». Certains acteurs ont plus de pouvoir et (ou), ici, plus d'autonomie que d'autres. Il faut distinguer, avec Crozier et Friedberg ${ }^{26}$, les deux. Si le pouvoir d'un acteur consiste à exercer un contrôle sur une ressource pertinente pour les autres, l'autonomie de l'acteur réside dans le fait qu'il contrôle la ou les ressources dont il a besoin. Elle correspond à la possibilité d'un acteur d'atteindre ses objectifs indépendamment des autres acteurs.

Ce que Friedberg (op. cit., p. 118) explicite ainsi : «Plus ego est capable par ses actions ou ses comportements d'apporter une solution plus ou moins permanente et plus ou moins adaptée à un problème qui pèse, menace, voire empêche la réalisation des projets cruciaux de alter, plus ce comportement de ego est important pour alter. $\{\ldots\}$ Reste qu'une ressource a plus ou mọns de pertinence pour l'action organisée ».

26. Michel Crozier et Erhard Friedberg, op. cit., p. 91-103. 
Nous proposons donc de formaliser cette notion d'autonomie ${ }^{27}$ comme la somme des enjeux qu'il place sur les relations qu'il contrôle :

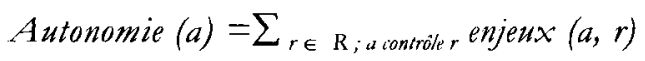

La dépendance d'un acteur est alors évaluée inversement comme la somme des enjeux qu'il place sur les relations qu'il ne contrôle pas :

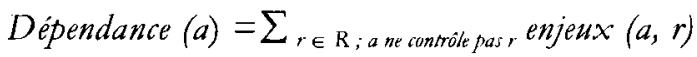

Dans le cas Trouville, nous obtenons les grandeurs suivantes :

Tableau 2. Pertinence des relations et l'autonomie des acteurs

\begin{tabular}{|l|c|c|c|c|}
\cline { 2 - 5 } \multicolumn{1}{c|}{} & $\begin{array}{l}\text { Stabilité } \\
\text { de } \\
\text { l'emploi }\end{array}$ & $\begin{array}{l}\text { Contenu } \\
\text { du travail }\end{array}$ & $\begin{array}{l}\text { Diffusion de } \\
\text { l'information }\end{array}$ & Autonomic \\
\hline $\begin{array}{l}\text { Enjeux } \\
\text { de la } \\
\text { secrétaire }\end{array}$ & 2 & 7 & 1 & 1 \\
\hline $\begin{array}{l}\text { Enjeux du } \\
\text { directeur }\end{array}$ & 1 & 2 & 7 & 3 \\
\hline Pertinence & 3 & 9 & 8 & \\
\hline
\end{tabular}

Nous constatons que les deuxième et troisième relations sont les plus pertinentes et que si le directeur est plus autonome que la secrétaire, leurs niveaux respectifs d'autonomie sont faibles. Cela exprime le fait qu'ils sont fortement dépendants l'un et l'autre, en l'occurrence l'un de l'autre. Mais qu'en est-il de leur pouvoir?

En prenant en compte les fonctions d'effet, nous pouvons revenir sur la notion du pouvoir qui est au centre de la sociologie de l'action

27 Dans une démarche différente, Emmanuel Lazega a montré que ces deux notions de pouvoir et d'autonomie pouvaient ètre opérationnalisées et quantifiées par l'analyse structurale. Emmanuel Lazega, Réseaux sociaux et structures relationnelles, Paris, PUF, coll. "Que sais-je?", 1998, p. 105-113. 
organisée. Considérons la grandeur $\max \left\{E_{f f e t}(a, r, \alpha)-E\right.$ ffet, $(a, r, \beta) ; a$, $\left.\beta \in\left[b_{\text {min }} b_{\text {max }}\right]\right\}$. Elle représente l'écart entre le meilleur et le pire solde que le contrôleur de la relation $r$ peut attribuer à l'acteur $a$. En la pondérant par l'enjeu que l'acteur a placé sur la relation $r$, nous proposons de la qualifier de pouvoir potentiel, en l'occurrence le pouvoir que l'acteur contrôlant la relation $r$ est en mesure d'exercer sur l'acteur $a$ par l'intermédiaire de cette relation que nous exprimons ainsi :

Pouvoir_potentiel( $r, a)=\operatorname{enjeu}(a, r) * \max \{\operatorname{Effet}(a, r, \alpha)-\operatorname{Effet}(a, r, \beta) ; a, \beta$ $\left.\in\left\{b_{\text {min }}, b_{\text {max }}\right]\right\}$

Mais nous pouvons aussi calculer ce powvoir potentiel cumulé d'un acteur $a$ sur un acteur $b$ à travers toutes les relations qu'il contrôle :

Pounoir_potentiel cumulé( $a, b)=\sum_{r \in R}$ aconsoler $r$ pounoir_potentiel( $\left.r, b\right)$

Enfin, il peut être intéressant de mesurer le pouvoir potentiel cumulé d'un acteur $a$ sur tous les autres acteurs par le biais de toutes relations qu'il contrôle :

Pouvoir_potentiel global $(a)=\sum_{b \in A}$ pouvoir_potentiel $(a, b)$

Ce pouvoir potentiel représente la mesure dans laquelle un acteur peut contribuer à la satisfaction d'un ou de plusieurs autres acteurs. Cette contribution dépendra de la manière dont il fixera effectivement les " termes de l'échange » dans l'intervalle qui lui est ouvert, ce que nous appelons l'état des relations. Ainsi à chaque état du système correspond un niveau de pouvoir actualisé, tel que nous l'avons défini antérieurement, qu'il faut différencier du pouvoir potentiel ou théorique. Le premier est fonction de l'état actuel du système, le second dépend de la seule structure du modèle et exprime le jeu des possibles.

Un grand nombre d'autres grandeurs susceptibles d'être interprétées dans les termes de la sociologie de l'action organisée peuvent être définies; nous ne nous y attardons pas. Dans le cas Trouville, celles que nous avons présentées prennent les valeurs suivantes: 
Tableau 3. Pouvoir potentiel et powvoir potentiel cumulé par relation et par acteur

\begin{tabular}{|c|c|c|c|c|c|c|c|c|c|}
\hline & \multicolumn{3}{|c|}{ Stabilité de l'emploi } & \multicolumn{3}{|c|}{ Contenu du travail } & \multicolumn{3}{|c|}{ Information sur TRO2 } \\
\hline & Effet & Enjeux & $\begin{array}{l}\text { Pouvoir } \\
\text { potentiel }\end{array}$ & Effet & Enjeux & $\begin{array}{l}\text { Pouvoir } \\
\text { potentiel }\end{array}$ & Effet & Enjeux & $\begin{array}{l}\text { Pouvoir } \\
\text { potentiel }\end{array}$ \\
\hline Directeur & $3 * x$ & 1 & 2,4 & $-3 * x^{2}$ & 2 & 9,6 & $10 * x$ & 7 & 70 \\
\hline Secrétaire & $10 * x$ & 2 & 16 & $-7 * x$ & 7 & 39,2 & $-2 *|x|$ & 1 & 1,4 \\
\hline $\begin{array}{l}\text { Pouvoir } \\
\text { cumulé }\end{array}$ & \multicolumn{3}{|c|}{18,4} & \multicolumn{3}{|c|}{48,8} & \multicolumn{3}{|c|}{71,4} \\
\hline $\begin{array}{l}\text { Pouvoir } \\
\text { global }\end{array}$ & \multicolumn{6}{|c|}{ Directeur : 67,2} & \multicolumn{3}{|c|}{ Secrétaire : 71,4} \\
\hline
\end{tabular}

À la lecture du tableau 3, nous constatons que la troisième relation contrôlée par la secrétaire est celle qui confère le plus de pouvoir global $(71,4)$. Mais comme le directeur maitrise les deux autres relations, il dispose d'un niveau de pouvoir global $(67,2)$ voisin de celui de la secrétaire. En d'autres termes, le directeur contrôle plus de relations que la secrétaire, mais cette dernière maitrise une relation qui impacte fortement la satisfaction du directeur. Il en résulte une situation où chacun dispose d'un niveau de pouvoir comparable sur l'autre, ce qui est conforme à l'analyse du système d'action que nous pouvons faire de manière plus traditionnelle. Elle explique que la proposition du directeur régional soit refusée par les deux. En effet, dans le nouveau jeu tel qu'il résulterait de la titularisation à plein temps de la secrétaire dans l'agence TRO1, les deux acteurs directement concernés disposeraient d'une capacité à atteindre leurs objectifs plus réduite. Le directeur perdrait la source d'information sur l'activité de TRO 2 que lui assure la présence à mi-temps de la secrétaire. Or, cette information est pour lui essentielle, c'est sur la relation qui lui est liée qu'il place principalement ses enjeux. Quant à la secrétaire, dans la nouvelle configuration, elle perdrait le moyen de peser sur la relation qui l'intéresse le plus, celle liée au contenu de son propre travail et à la manière dont il est contrôlé. En coopérant, les deux acteurs s'assurent mutuellement un niveau de 
satisfaction dont rien ne leur assure qu'ils le conserveraient dans une nouvelle structure de jeu.

Mais pour confirmer cette analyse encore faut-il montrer que l'état du jeu social tel qu'il se déroule se stabilise dans une situation assurant à chacun un niveau de satisfaction acceptable, c'est-à-dire une situation voisine d'un optimum de Pareto. C'est l'objet de la simulation que nous verrons dans la section suivante La formalisation des contraintes permet de définir certaines quantités qui sont susceptibles d'être interprétées comme des propriétés significatives du système d'action concret. Ainsi, pouvons-nous proposer une formalisation de l'influence, de la pertinence d'une ressource et du pouvoir, de l'autonomie et de la dépendance d'un asteur.

\section{Simulation du comportement des acteurs}

Pour comprendre ce que nous simulons, il faut distinguer ce que nous désignons comme étant les dimensions fonctionnelle et structurelle d'un système. Cela étant fait, nous pourrons présenter un dispositif d'apprentissage permettant de simuler la dimension fonctionnelle du comportement des acteurs, sa dimension structurelle restant à étudier.

4.1. Dimensions fonctionnelle et structurelle du comportement de l'acteur

Le formalisme de modélisation que nous venons d'exposer permet de distinguer, dans un système d'action concret, ce qui relève de sa structure - ses éléments constitutifs, leurs relations et les opérations auxquelles ils peuvent donner lieu -, et ce qui relève de son état dont l'évolution accompagne l'accomplissement des finalités du système. La structure d'un système d'action concret se décrit en termes d'acteurs, de relationstransactions fondées sur des ressources, d'enjeux placés sur ces relations et de fonctions d'effet et de contraintes sur le contrôle. Quant à l'état du système d'action, il se décrit en termes d'état des relations. Pour chaque acteur, les soldes qu'il reçoit en fonction de cet état des relations correspondent aux moyens d'action à sa disposition.

Cela nous amène à distinguer deux dimensions dans l'action d'un acteur qui cherche à conforter son pouvoir : une dimension structurelle qui agit sur la structure du système et une dimension fonctionnelle qui agit sur son état. 
La dimension structurelle de l'action est la part qui contribue à la construction de l'organisation du système d'action concret, à l'établissement des règles du jeu social et qui donc consiste, selon notre formalisation, à agir sur les éléments qui constituent sa structure : acteurs, relations, contraintes et enjeux. Quant à la dimension fonctionnelle de l'action d'un acteur, c'est celle qui assure le fonctionnement régulier du système et fait évoluer son état de façon synchronique; elle concourt à la réalisation des objectifs immédiats de l'acteur. Cette dimension fonctionnelle s'exerce à des règles du jeu constantes, sans changement ni dans les objectifs, c'est-à-dire des enjeux, ni dans les moyens d'actions, c'est-à-dire dans les fonctions d'effet des relations et leurs contraintes. Évidemment, dans l'action concrète d'un acteur, ces deux dimensions sont indissociables l'une de l'autre : chaque acte comporte une composante structurelle et une composante fonctionnelle, dans une proportion spécifique aux circonstances de la réalisation de cet acte. Pour autant, nous ne cherchons pas à modéliser les modalités pratiques des actes et ne nous intéressons qu'à leurs effets ; ces effets sur les deux dimensions structurelle et fonctionnelle étant bien disjoints, nous avons la possibilité, en ce qui concerne la simulation, de modéliser l'action des acteurs par des mécanismes spécifiques à chacune de ces dimensions. Nous avons modélisé l'action «fonctionnelle» des acteurs et travaillons à la modélisation de l'action «structurelle", mais cette dernière nécessite un modèle du changement organisationnel qui n'est pas présent dans la sociologie de l'action organisée.

Dans la dimension fonctionnelle du comportement des acteurs, toute action consiste à exercer le contrôle sur une relation que l'on maîtrise, c'est-à-dire à manipuler l'état de cette relation (les " termes de l'échange ") et par là la valeur des soldes attribués aux participants tout en restant dans les limites imposées par les contraintes de cette relation. Ce faisant, chaque acteur met en ouvre des stratégies visant à obtenir la plus grande capacité à atteindre ses objectifs que nous exprimons par le terme satisfaction. Quand les niveaux de satisfaction des acteurs se stabilisent lors d'une simulation, c'est que chacun a trouvé les valeurs des «termes de l'échange » qui lui conviennent et qu'il va maintenir. En d'autres termes, certaines zones de l'espace des états du système constituent des attracteurs qui traduisent l'existence d'une régulation du système modélisé. Or, la régulation, qui permet qu'un système d'action concret puisse exister et être repéré par le sociologue, est bien le 
phénomène dont la sociologie de l'action organisée s'assigne pour objectif la mise en lumière. Si cette régulation produite par la simulation correspond à un état du système modélisé qui peut ètre interprété sociologiquement comme étant conforme aux résultats de l'étude empirique, alors nous considérons avoir réussi à simuler le fonctionnement du système d'action concret étudié. Pour comprendre le fonctionnement des simulations, il faut bien distinguer, d'une part l'espace des états du système et, d'aurre part, l'espace des satisfactions des acteurs ainsi que l'interaction entre les deux : au cours d'une simulation, les acteurs modifient l'état du système (en agissant sur l'état des relations qu'ils contrôlent) en fonction de leur satisfaction courante, et réciproquement l'état du système détermine le niveau de leur satisfaction. Le système est régulé lorsque cette boucle est stationnaire, chacun des acteurs ayant une satisfaction qui l'incite à ne pas modifier l'état des relations qu'il contrôle. Mais la simulation nécessite de doter nos acteurs d'un modèle de rationalité.

\subsection{Un modèle par apprentissage de la dimension fonctionnelle du comportement des acteurs}

L'hypothèse de rationalité de la sociologie de l'action organisée conduit à fonder ce comportement sur le cycle de base :

1. percevoir l'état du système et notamment sa propre satisfaction,

2. décider de l'action à entreprendre,

3. exécuter cette action, et ce, jusqu'à ce que le jeu se stabilise, c'est-à-dire aucun acteur ne souhaite plus modifier l'état des relations qu'il contrôle; il accepte sa propre satisfaction et celle des autres. Concrètement, il se comportera dans ses transactions avec les autres acteurs de façon à leur octroyer les soldes correspondant à l'état actuel des relations qu'il contrôle, le jeu social est régulé. Le principe de la simulation consiste donc à doter les acteurs d'une rationalité leur permettant, à partir d'un état arbitraire du système, de découvrir collectivement un état (ou l'un des états) pour lequel le système est régulé. Demeure la question de l'état du système d'action vers lequel nous souhaitons que la simulation converge. Fondamentalement, les organisations sociales fonctionnent, et ce parce que les acteurs sociaux sont globalement coopératifs tant que ce n'est pas à leur désavantage manifeste ; la " grève du zèle » reste un 
comportement de crise. Nous cherchons donc à doter les acteurs d'un comportement tel que le système converge vers des états qui soient, aux variations stochastiques près, des optima de Pareto (ce qui ne préjuge pas de l'immense intérêt de trouver des modèles de rationalité des acteurs tels que le « jeu social » converge vers des équilibre de Nash, ou les états élitistes ou égalitaires que nous évoquons en conclusion). De plus, si l'algorithme qui modélise le comportement d'un acteur social est bien un domaine relevant de la compétence spécifique de l'informaticien, les conséquences des choix opérés ont des conséquences sur les résultats des simulations et le sociologue ne peut pas s'en désintéresser : la logique de cet algorithme et les valeurs de ses paramètres doivent pouvoir être mises en relation avec la nature même du comportement social.

L'opération centrale, le choix de l'action à réaliser, peut être implantée de différentes façons. Une approche cognitive pourrait utiliser une logique des croyances de profondeur limitée $e^{28}$ permettant à chaque acteur de raisonner pour identifier explicitement les acteurs dont il dépend ${ }^{29}$ et se comporter en conséquence. Une autre approche, de type résolution de problème, consisterait à utiliser des algorithmes génétiques, mais sans que nous puissions alors interpréter le processus de régulation en termes de compétences cognitives des acteurs sociaux. C'est une autre approche que nous avons implantée dans l'environnement SocLab ${ }^{30}$. Il repose sur l'apprentissage de règles comportementales par essais-erreurs et par renforcement des règles en fonction des

28 Herbert Simon, Sciences des systemes, sciences de l'artificiel, Paris, Dunod, [1981] 1991.

29 Jaime Sichman, Rosaria Conte, Cristiano Castelfranchi et Yves Demazeau, «A social reasonning nechanism based on dependence networks ", Proceedings of the $3^{\text {rit }}$ Italian Workshop on Distributed Artificial Intelligence (ECAI), Amsterdam, 1994, p. 173-177.

30 Matthias Mailliard, Frédéric A mblard, Christophe Sibertin-Blanc et Pascal Roggero, "Cooperation is not always so simple to learn..." dans TeranoTakao, Hajime Kita, Hiroshi Deguchi et Kyoichi Kijima (dir.), Agent-based Approaches in Economic and Social Complex Systems IV, Springer, 2005, p. 147-154; Christophe Sibertin-Blanc, Frédéric Amblard et Matthias Mailliard, «A coordination framework based on the Sociology of Organized Action ", dans Olivier Boissier et al. (dir.), Coordination, Organizations, Institutions and Norms in Multi-Agent Systems, vol. 3913, Lecture Notes in Comuter Sciences, Springer, 2006, p. 3-17. 


\section{NPSS, VOLUME 3, NUMERO 2, 2008}

résultats qu'elles produisent ${ }^{31}$. Cette approche présente deux avantages par rapport à l'approche cognitive : elle se contente d'un modèle global du système d'action concret, alors que l'approche cognitive requiert d'expliciter la représentation que chacun des acteurs se fait du système en question. De plus, elle fait très peu d'hypothèses sur les aptitudes requises pour être un acteur dans un système d'action concret, notamment, elle ne préjuge pas de ce que seraient des règles explicites du comportement social que les acteurs appliqueraient.

Ce système de règles permet donc aux acteurs d'apprendre des stratégies par renforcement de règles comportementales qu'ils inventent au hasard. Schématisons : nos acteurs agissent de manière incrémentale sur "les termes de l'échange ", c'est-à-dire l'état des relations qu'ils contrôlent et ils sont renforcés dans cette action si leur satisfaction augmente. Il y a donc un facteur «técompense »dont résulte ce que l'on va appeler la force de la règle. La règle est du type : dans tel état du système, je modifie de telle manière l'état des relations que je contrôle. Le processus d'apprentissage de l'acteur consiste donc à se constituer une base de règles que nous pouvons considérer comme une forme de mémoire. Cette base de règles est actualisée de manière continue selon un mécanisme associant au facteur récompense, qui augmente ou affaiblit la force de la règle, un facteur d'oubli. Ce facteur d'oubli diminue un peu la force de la règle quand elle n'est pas appliquée ct la supprime - elle disparaît de la base de règles - lorsque sa force est négative. Dans le cas, inévitable au début de la simulation, où aucune règle n'est applicable (ou connue), l'acteur joue au hasard, il invente une règle. Il reste que l'évaluation de la pertinence d'une règle suppose que l'acteur puisse comparer l'état courant du système avec celui enregistré dans les règles de sa base. En d'autres termes, qu'il puisse, dans une situation du système qu'il n'a jamais rencontrée, regarder s'il a connu des situations proches et considérer les règles qui s'y sont appliquées. Il reste à déterminer cet espace. Nous l'avons représenté sous la forme d'un disque ou d'une hyper-boule suivant le nombre de relations que compte le système, dont la taille dépend de la valeur du paramètre rayon. Ce rayon fixe donc l'espace des situations considérées par l'acteur comme étant

\footnotetext{
John Holland et Judith Reitman, “Cognitive system based on adaptive algorithms », dans Pattern Directed Inference Systems, New York, Academ ic Press, 1978, p. 313-329 et Olivier Sigaud, "Les systèmes de classeurs : un état de l'art ", Revue d'Intelligence Artificielle, Hermès Publisher, 2007, p. 75-106.
} 
proches, et c'est dans cet espace qu'il va rechercher la règle la plus forte et l'appliquer. Ce paramètre, qui peut s'interpréter comme la capacité de discrimination de l'acteur, a donc une influence considérable sur son comportement. Ainsi, si on lui fixe une valeur élevée, l'acteur va plus exploiter les règles déjà connues qu'explorer de nouvelles règles et, inversement, si la valeur du rayon est faible. Cette problématique exploration-exploitation ${ }^{32}$ est très féconde pour le sociologue qui peut ainsi tenter de caractériser de manière formelle des dimensions très significatives du comportement des acteurs telle que le conformisme, le ritualisme, l'aventurisme, etc. Il y a là une vraie heuristique sociologique. Pour obtenir des résultats de simulation intéressants, il s'est avéré nécessaire de considérer que notre acteur possédait une aptitude à évaluer socialement sa satisfaction. En d'autres termes, s'il peut comparer des niveaux de satisfaction successifs, il est aussi en mesure de porter un jugement plus général sur sa satisfaction présente par rapport à ses attentes, à ce qu'il peut attendre plus globalement du jeu. Ces attentes, évidemment, existent dans le monde social dans lequel l'acteur juge sa situation présente non seulement par rapport à celle qui précède, mais aussi par rapport à des attentes subjectives plus globales comme le montrent bien, à leur manière, les théories de la frustration relative ${ }^{33}$. Cela nous amène à introduire dans l'algorithme de chaque acteur un seuil de satisfaction qui évolue au fur et à mesure. Cette évolution est à la baisse en partant d'une situation initiale que nous avons choisie comme étant sa satisfaction maximale. La rapidité de cette évolution est fonction, d'une part, de la distance entre ce seuil et sa satisfaction courante (à la façon d'un élastique) et, d'autre part, d'un coefficient lié à la complexité du jeu social, c'est-à-dire le nombre d'acteurs et de relations. Ainsi, le comportement de l'acteur peut être différent selon qu'il estime se trouver ou pas dans une situation acceptable au regard de ses attentes. Dans le premier cas, notre acteur aura un comportement marqué par l'exploitation, dans le second, il sera plus explorateur. En d'autres termes, il change peu, voire pas, une situation qui le satisfait par rapport à ses

\footnotetext{
32 James G. March, «Exploration and Exploitation in Organizational Learning », Organization Science, vol. 2, n ${ }^{\circ} 1,1991$, p. 71-87.

33 Ted Robert Gurr, W by men rebel?, Princeton, Princeton University Press, 1970, et Jean Kellerhals, Josette Coenen-Huther et Marianne Modak, Figures de l'équité. L a construction des normes de justice dans les groupes, Paris, PUF, coll. "Le sociologue ", 1992.
} 
attentes. Dans le cas symétrique, au contraire, il tente de changer la situation, et le jeu sera régulé lorsque chaque acteur aura une satisfaction au moins égale à son seuil de satisfaction.

La propension à l'exploration ou à l'exploitation amène à moduler la récompense et l'oubli des règles, ainsi que l'intensité des actions, c'est-àdire l'importance des déplacements de l'état des relations. Plus précisément, en mode exploratoire une récompense positive nécessite un gain de satisfaction important, l'oubli est plus rapide et les actions sont plus intenses. Les résultats de simulations présentées à propos du cas Trouville intègrent ce modèle de rationalité. 11 est maintenant nécessaire de proposer une application de ce méta-modèle.

\subsection{Simulation du cas Trouville}

L'espace des états du système présente ici trois dimensions puisqu'il y a trois relations. Il peut être décrit analytiquement et il définit toutes les configurations possibles du système. Ainsi pour chaque triplet définissant les "termes de l'échange " des trois relations, nous pouvons calculer les valeurs de grandeurs telles que le pouvoir et la satisfaction de chaque acteur, ou la satisfaction globale. Tous les points dans cet espace à trois dimensions existent mathématiquement, mais tous n'ont pas de sens ou de plausibilité sociologiques. Il faut donc identifier les situations remarquables dans cet espace, en d'autres termes des situations que nous pouvons interpréter sociologiquement. C'est que nous avons fait dans le tableau 3 en ne retenant que la grandeur Satisfaction que nous jugeons comme étant la plus synthétique.

Ainsi avons-nous identifié des triplets significatifs qui définissent des situations sociologiquement interprétables. La satisfaction maximale de la secrétaire $(27,6)$ est obtenu pour le triplet $(-0,4 ; 0,4 ; 0)$ mais la satisfaction du directeur est alors négative $(-8,4)$. Cette situation n'est pas vraisemblable, et la satisfaction globale qu'elle produit $(19,2)$ est très éloignée du maximum théorique. Pour le directeur, c'est le triplet $(0$; $0,4 ; 0,7)$ qui lui procure la meilleure satisfaction $(50,2)$, mais avec une très faible satisfaction de la secrétaire $(6,6)$ et toutefois une meilleure satisfaction globale que dans le cas précédent. Enfin, dernière situation remarquable considérée ici, la meilleure satisfaction globale $(74,4)$, est associée à des niveaux de satisfaction élevés pour chacun des acteurs. Elle correspond au triplet $(-0,4 ; 0,4 ; 0,7)$. Cette situation est aussi un 
optimum de Pareto, et c'est la plus vraisemblable au regard du cas empirique : elle correspond à la coopération entre les acteurs, chacun mettant du sien pour que l'autre fasse de même.

Tableau 4. Calcul analytique de la Satisfaction des acteurs dans trois états remarquables

\begin{tabular}{|l|l|c|c|c|}
\cline { 2 - 4 } \multicolumn{2}{c|}{} & $\begin{array}{l}\text { Satisfaction } \\
\text { maximale } \\
\text { de la } \\
\text { secrétaire }\end{array}$ & $\begin{array}{l}\text { Satisfaction } \\
\text { maximale } \\
\text { du directeur }\end{array}$ & $\begin{array}{l}\text { Satisfaction totale } \\
\text { maximale }\end{array}$ \\
\hline $\begin{array}{l}\text { État des } \\
\text { relations }\end{array}$ & $\begin{array}{l}\text { Contenu du } \\
\text { travail }\end{array}$ & $-0,4$ & 0 & $-0,4$ \\
\cline { 2 - 5 } & $\begin{array}{l}\text { Stabilité } \\
\text { emploi }\end{array}$ & 0,4 & 0,4 & 0,4 \\
\cline { 2 - 5 } & $\begin{array}{l}\text { Info sur } \\
\text { TRO2 }\end{array}$ & 0 & 0,7 & 0,7 \\
\hline \multirow{5}{*}{ Satisfaction } & Directeur & $-8,4$ & 50,2 & 48,2 \\
\cline { 2 - 5 } & Secrétaire & 27,6 & 6,6 & 26,2 \\
\hline & $\begin{array}{l}\text { Satisfaction } \\
\text { globale }\end{array}$ & 19,2 & 56,8 & 74,4 \\
\hline
\end{tabular}

Une fois ce cadre analytique fixé, nous explorons par la simulation le comportement des acteurs en regardant si le jeu virtuel tend vers une zone particulière de l'espace. La figure 4 montre les résultats d'une telle simulation ayant convergé au bout de 450 pas de simulation. La forme erratique des courbes tient à l'apprentissage des acteurs. 
Figure 4. Exemple de simulation du cas Trouville (abscisses : pas de simulation; ordonnées : état des relations/satisfaction des acteurs)

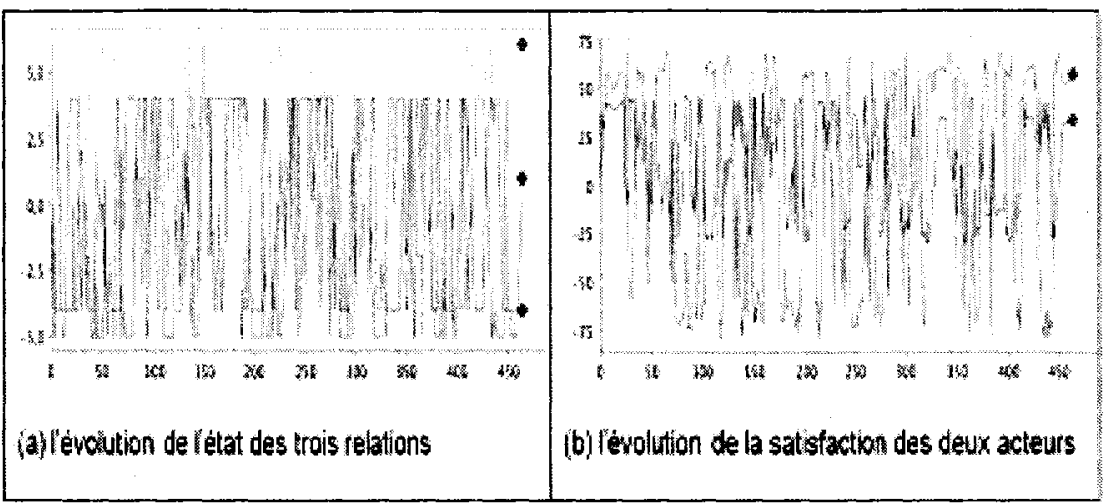

L'expérience de simulation sur le modèle confirme donc l'analyse empirique réalisée en convergeant vers la coopération ${ }^{34}$.

Tous ces résultats numériques doivent être considérés avec beaucoup de prudence avant qu'ils n'autorisent une interprétation sociologique significative. En premier lieu, les échelles de valeur sont arbitraires; aussi chaque valeur prise isolément n'a aucune signification, elle n'en a que relativement aux autres. Ensuite, l'écart entre ces valeurs doit être important - de l'ordre de 20 à $30 \%$, ce qui est le cas dans notre exemple - pour être considéré comme significatif. En effet, les paramètres sont fixés d'après une étude sociologique qualitative qui ne requiert pas, habituellement, le niveau de précision ici exigé par la quantification. Il y a donc une forme d'arbitraire - certes raisonné - dans la fixation des valeurs numériques des paramètres du modèle. De plus, les formules proposées pour évaluer le pouvoir ou la satisfaction des acteurs ne résultent pas d'un argument formel, mais sont fondées sur une formalisation, nécessairement simplificatrice des concepts de la socio-

34 Pascal Roggero et Christophe Sibertin-Blanc, «Approche complexe de l'action organisée : formalisation et simulation orientée agents des systèmes d'action concrets ", Colloque Systemes complexes en SHS, Centre international culturel de Cerisy, Cerisy-La-Salle, 26 mai 2007 - 2 juin 2007 , http:/ / www.cciccerisy.asso.fr/systemescomplexes07.html, consulté le 25 septembre 2007. 
logie de l'action organisée, que la formulation primitive dans le seul langage naturel rend difficile à synthétiser formellement.

\section{Conclusion : les raisons de continuer}

Il y a, assurément, beaucoup de limites dans l'exemple proposé et, plus généralement, dans la démarche que nous avons présentée. Il ne faut pas se les cacher et essayer de les examiner lucidement. Mais, pour autant, cette démarche qui commence à faire ses premiers pas, présente, à nos yeux, une indéniable fécondité.

\section{Des limites considérables}

Il y a celles qui relèvent de notre exemple sur lesquelles nous irons vite et puis celles plus fondamentales qui concernent l'ensemble de la démarche.

Notre cas est évidemment imparfait à bien des égards. Ainsi avonsnous choisi la prise en compte des deux seuls acteurs principaux - le directeur et la secrétaire - sans considérer les autres. On pourrait, à juste titre, nous le reprocher. Encore une fois notre volonté était d'aller au plus simple pour éprouver notre méta-modèle. D’autres critiques pourraient assurément être faites, mais elles nous renvoient à celles que la démarche peut susciter

Si la formalisation en elle-même nous apparaît assez solidement construite et traduire fidèlement, dans l'ensemble, les concepts de la sociologie de l'action organisée, d'importants problèmes apparaissent dans son application empirique. Parmi ceux-ci figure la question déjà signalée de l'évaluation quantitative de paramètres qui sont généralement évalués qualitativement. Une partie de la réponse réside dans l'adoption ${ }^{35}$ d'une logique floue pour travailler avec un niveau de précision moins draconien dans l'évaluation des paramètres et induisant, pour l'utilisateur du modèle, un arbitraire moins important. En effet, la logique floue permet de donner aux paramètres une plage de valeurs au

35 Déjà engagée : Sandra Sandri, Christophe Sibertin-Blanc et Vicenç Torra, «A Multicriteria Fuzzy System Using Residuated Implication Operators and Fuzzy Arithmetic », dans Yasuo Narukawa, Torra Vincenç et Yukio Yoshida (dir.), IEEE International Conference on Penasive Systems (MAPS 2007), Kitakyushu, Japon, 16/08/2007-18/08/2007, vol. 4617, Springer-Verlag, LNAI, 2007, p. 57-67. 
lieu d'une valeur fixe définitive ${ }^{36}$ et d'alléger cette contrainte de quantification. Il reste que cette quantification peut être considérée comme un réductionnisme que l'on peut juger assez éloigné du "réalisme " sociologique et de la pensée complexe. Pour un sociologue qui se pique de complexité, cela peut faire beaucoup. À cette objection fondamentale, nous pouvons répondre en évoquant l'intérêt de la démarche. Pour le moment, cette démarche n'a pour ambition ni de décrire le social avec réalisme, ni d'en prévoir l'évolution, ni de l'enfermer dans un formalisme étroit. Non, nous avons bien conscience de travailler sur des représentations et nous cherchons à en assurer la cohérence en les fondant assez précisément. Les limites viennent surtout, de notre point de vue, du caractère récent et asséz novateur de notre démarche : nous en sommes encore aux balbutiements. Ainsi, par exemple, nous ne savons simuler un système d'action concret que dans une dynamique "fonctionnelle ", c'est-à-dire à éléments structurels fixés. Nos acteurs manipulent les "termes de l'échange " à contraintes, relations et enjeux constants. Nous travaillons aujourd'hui à concevoir la dynamique "structurelle » du modèle. Par ailleurs, nous n'intégrons pas non plus l'environnement. Grave péché pour des systémiciens! Là aussi, nous comptons bien y travailler en creusant notamment la complémentarité possible avec l'approche des réseaux suciaux. De même, nous raffinons de jour en jour le dispositif d'apprentissage encore bien fruste dont nous dotons nos acteurs. Bref, les pistes de travail ne manquent pas, le temps et les moyens humains beaucoup plus. On pourrait juger qu'après plusieurs années, notre collaboration n'a pas abouti à quelque chose de vraiment satisfaisant. Une telle perception manquerait une donnée essentielle, absolument nécessaire à tout travail interdisciplinaire et, plus encore, transdisciplinaire, le temps de l'apprentissage mutuel. Entre informaticiens et sociologues, la distance épistémologique et méthodologique est grande. Nous avons donc travaille pour le long terme en consentant un véritable investissement cognitif qui nous assure que les bases de notre travail commun sont solides. Et si nous tenons à ce projet, c'est qu'il nous apparait fécond. Nigel Gilbert écrit à propos des difficultés induites par le travail de simulation:

Paradoxically, one of the main advantage of simulation is it is hard to do. To create a useful simulation model, its theorical presuppositions need to have been thought through with great clarity. Every relationship to be modelled has to be

36. Bernadette Bouchon-Meunier, La logique flout, Paris, PUI , coll. «Que sais-je ?", 1993. 
specified exactly and every parameter has to be given a value, for otherwise it will impossible to run the simulation ${ }^{37}$.

Ce fut effectivement difficile à faire et si notre formalisation présente des limites, elle a un réel intérêt.

\section{Une fécondité protéiforme}

Commençons par l'empirique. Nous en saurons plus quand nous aurons réalisé une enquête destinée à rendre compte d'un « terrain » et à nourrir explicitement le méta-modèle. Mais, déjà, il est possible de tracer quelques pistes pour l'orientation de l'investigation empirique. L'identification des ressources, des relations et des contrôleurs fait partie du travail habituel d'un sociologue de l'organisation. Il faut cependant insister sur la nécessité de bien distinguer ressource et relation ce que ne fait pas suffisamment, nous semble-t-il, Friedberg. Plus problématique est la collecte de données les plus précises possibles sur les « termes de l'échange » entre les acteurs et sur les contraintes qui pèsent sur eux. On se contente souvent d'une appréciation, somme toute assez vague, du type : "l'acteur $x$ fixe les termes de l'échange » ou " est dominant dans l'échange ». Pour noutrir notre méta-modèle, il nous faut aller plus en profondeur. Par exemple, en interrogeant systématiquement les acteurs et leurs pratiques sur la manière dont ils réagissent précisément aux différents comportements de leurs partenaires dans la relation jusqu'à imaginer dans le détail des situation auxquelles il est demandé à l'acteur de dire comment il les considérerait et comment il y réagirait. Dans notre exemple, il nous aurait été nécessaire d'interroger la secrétaire sur son jugement et sa réaction si le directeur la contrôlait un peu ou beaucoup plus, etc. L'idée étant aussi de repérer les contraintes, il est important de saisir au-delà des routines jusqu'où on peut ne pas aller trop loin en identifiant ce qui est acceptable, peu acceptable et intolérable. Le même problème se pose pour la détermination précise des enjeux. Il faut, en l'occurrence, identifier les relations dans lesquelles l'acteur est engagé sans se limiter à la plus évidente et lui demander de préciser l'importance qu'il leur accorde en utilisant une échelle d'évaluation suffisamment détaillée pour ensuite, par comparaison, en

37 Nigel Gilbert, «Quality, quantity and the third way », Département de sociologie de l'Université de Surrey, no 160, 2004, p. 4, http://www.coc.surrey.ac.uk/staff/ngilbert/ngpub/index.html, consulté le 18 février 2008. 
déterminer les enjeux. Nous pouvons aussi envisager, bien que la pratique n'en soit pas toujours aisée en milieu organisationnel, l'utilisation de questionnaires élaborés après un travail exploratoire permettant de disposer de données en nombre et facilement traitables. Ensuite, au fur et à mesure de nos avancées, il est sans doute souhaitable que nous élaborions un guide d'entretien type voire un questionnaire type, dédiés à l'évaluation des paramètres du méta-modèle. Cela semble indispensable si, comme nous le souhaitons, nous créons un outil à usage public.

Il est dans nos perspectives de constituer un atelier de simulation qui; étant assorti d'un guide méthodologique, pourrait rendre possible une utilisation du méta-modèle dans différents cadres : scientifique, pédagogique et professionnel. Pour l'enseignement de la sociologie des organisations, le méta-modèle avait fait l'objet d'une utilisation devant un public de master recherche. L'avantage de cet outil est de présenter une formalisation qui donne aux concepts utilisés un contenu explicite et quantifiable. À ce titre, il participe d'une objectivation des fondements théoriques, des attendus méthodologiques et des résultats de la démarche sociologique. On passe ainsi d'un niveau chargé d'implicite ou d'imprécision théorique, se contentant souvent d'approximations empiriques et de résultats quelquefois rhétoriques, à une démarche plus exigeante. Comme nous l'avons vu, la démarche ne supprime évidemment pas l'arbitraire et la subjectivité de l'analyste, mais elle les encadre plus strictement, lui impose de devoir mieux les justifier et ainsi d'en prendre une conscience plus claire. Elle ne saurait se substituer aux méthodes existantes - qualitatives comme quantitatives -, le plutalisme est de rigueur en la matière. Elle ajoute un registre à la panoplie méthodologique du sociologue ${ }^{38}$.

Sur le plan professionnel, les perspectives apparaissent plus lointaines, mais ne sont pas pour autant inexistantes. En effet, beaucoup de cabinets de consultants utilisent la sociologie de l'action organisée pour établir des diagnostics organisationnels et préconiser des "solutions" à des difficultés. Or, ces diagnostics ne disposent généralement pas d'autres moyens de vérifier leur validité en dehors de la réception des résultats par les acteurs. L'utilisation raisonnée du méta-modèle pourrait représenter une manière d'éprouver les résultats empiriques obtenus en 
les confrontant aux résultats simulés. Un tel usage ne devrait cependant pas se pratiquer avec l'illusion qu'on accéderait ainsi à une "vérité ", tout juste à une connaissance peut-être mieux fondée, mieux contrôlée et plus partagée. Cet usage professionnel, hypothétique pour l'instant, pose cependant des questions éthiques. Encore une fois, il ne saurait s'agir d'une forme de "scientisation » du social ou de l'organisationnel, aucun usage de ce type, qui prétendrait s'appuyer sur cet outil pour prétendre à une scientificité devant par nature s'imposer, ne pourrait avoir l'agrément de ses concepteurs. Il est donc nécessaire de travailler à la meilleure diffusion de l'outil pour qu'il ne devienne pas l'apanage d'une catégorie particulière.

Mais nous n'en sommes pas là. Notre méta-modèle doit être éprouvé sur un ensemble de cas suffisamment nombreux pour en tester la robustesse et le pouvoir d'expression. Cela se fera dans les mois à venir. Pour le moment, insistons sur ce qu'en l'état, il nous permet de faire. Un des aspects de la formalisation et de la simulation, dès lors qu'elles se fondent, comme dans notre cas, sur un corpus théorique identifié se présente comme une épreuve de cohérence pour le dit corpus et, corrélativement, comme un puissant moyen critique stimulant l'imagination théorique du sociologue.

\section{Bibliographie}

Amblard, Frédéric et Denis Phan, Modélisation et simulation multi-agents. Applications aux Sciences de l'Homme et de la Société, Hermès Science Publication, 2006, 446 p.

Bouchon-Meunier, Bernadette, La logique floue, Paris, PUF, coll. «Que sais-je ? », 1993, $128 \mathrm{p}$.

Crozier, Michel et Erhard Friedberg, L'Acteur et le Systeme. Contraintes de l'action collective, Paris, Seuil, 1977, 500 p.

Ferber, Jacques, Les systèmes multi-agents. Vers une intelligence collective, Paris, InterEditions, [1995] 2007, $522 \mathrm{p}$.

Friedberg, Erhard, Le pouvoir et la règle. Dynamiques de l'action organisée, Paris, Seuil, coll. «Sociologie », 1993, 405 p.

Gilbert, Nigel, "Quality, quantity and the third way », Département de sociologie de l'Université de Surrey, n" 160, 2004, p. 4, http://www.coc.surrey.ac.uk/staff/ngilbert/ngpub/index.html, consulté le 18 février 2008. 
Gilbert, Nigel, "Using computer simulation to study social phenomena ", Bulletin de Méthodologie Sociologique, $\mathrm{n}^{\circ} 47,1995$, p. 99-111.

Gurr, Ted Robert, Why men rebel?, Princeton, Princeton University Press, 1970, 421 p. Holland, John et Judith Rejtman, "Cognitive system based on adaptive algorithms ", dans Pattern Directed Inference Systems, New York, Academic Press, 1978, p. 313 329.

Kellerhals, Jean, Josette Coenen-Huther et Marianne Modak, Figures de l'équité. La construction des normes de justice dans les groupes, Paris, PUF, coll. "Le sociologue », $1992,225 \mathrm{p}$.

Lazega, Emmanuel, Réseaux sociaux et struciures relationnelles, Paris, PUF, coll. «Que saisje? ", $1998,128 \mathrm{p}$.

Mailliard, Matthias, Frédéric Amblard, Christophe Sibertin-Blanc et Pascal Roggero, "Cooperation is not always so simple to learn... », dans TeranoTakao, Hajime Kita, Hiroshi Deguchi et Kyoichi Kijima (dir.), Agent-based Approacbes in Ficonomic and Social Complex Systems IV, Springer, 2005, p. 147-154.

March, James G., "Exploration and Exploitation in Organizational Learning ", Organization Science, vol. 2, $\mathrm{n}^{\circ} 1,1991$, p. 71-87.

Programme européen Modélisation de la complexité-Association pour la pensée complexe, Lexique, 2007, http://www.mcxapc.org, consulté le 25 juin 2007.

Roggero, Pascal, "Pour une sociologie d'après La Métbode ", Communications, $\mathrm{n}^{\circ} 82$, 2008, p. 143-160.

Roggero, Pascal et Christophe Sibertin-Blanc, "Approche complexe de l'action organisée : formalisation et simulation orientée agents des systèmes d'action concrets ", Collnque Systemes complexes en SHS, Centre international culturel de Cerisy, Cerisy-La-Salle, 26 mai 2007 - 2 juin 2007 , http://www.cciccerisy.asso.fr/systemescomplexes07.html, consulté le 25 septembre 2007 .

Roggero, Pascal et Christophe Sibertin-Blanc, «Pour une formalisation de la Sociologie de l'Action Organisée ", Communication au XVII Congrès international des sociologues de langue francaise, Tours, 5-9 juillet 2004.

Sainsaulieu, Renaud, L'identité au trayall, Paris, Presses de Science Po, [1977] 1988, $480 \mathrm{p}$.

Sandri, Sandra, Christophe Sibertin-Blanc et Vicenç Torra, «A Multicriteria Fuzzy System Using Residuated Implication Operators and Fuzzy Arithmetic ", dans Yasuo Narukawa, Torra Vincenç et Yukio Yoshida (dir.), IEEE International Conference on Pervasive Systems (MAPS 2007), Kitakyushu, Japon, 16/08/200718/08/2007, vol. 4617, Springer-Verlag, LNAI, 2007, p. 57-67.

Sibertin-Blanc, Christophe, Frédéric Amblard et Matthias Mailliard, "A coordination framework based on the Sociology of Organized Action ", dans Olivier Boissier et al. (dir.), Coordination, Organizations, Institutions and Norms in Multi-1gent Systems, vol. 3913, Lecture Notes in Comuter Sciences, Springer, 2006, p. 3-17.

Sibertin-Blanc, Christophe, Matthias Mailliard et Pascal Roggero, «Un modèle de rationalité limitée des acteurs sociaux ", dans Vincent Chevrier et Marc-Philippe Huguet (dir.), Systèmes multi-agents : articulation entre l'individuel te le collectif, Paris, Hermès Lavoisier, 2006, p. 95-98. 


\section{PASCAL ROGgERO... / QUANO DES SOCIOLOGUES RENCONTRENT...}

Sichman, Jaime, Rosaria Conte, Cristiano Castelfranchi et Yves Demazeau, "A social reasonning nechanism based on dependence networks ", Proceedings of the $3^{\text {rd }}$ Italian Workshop on Distributed Artificial Intelligence (ECAI), A msterdam, 1994, p. 173-177.

Sigaud, Olivier, "Les systèmes de classeurs : un état de l'art », Revue d'Intelligence Artificielle, Hermès Publisher, 2007, p. 75-106.

Simon, Herbert, Sciences des systemes, sciences de l'artificiel, Paris, Dunod, [1981] 1991.

Smets, Patrick, L'agence Travel-Tours (Troutille), 2004, http://homepages.ulb.ac.be/ psmets1/travel.pdf, consulté en juin 2004. 